\title{
On Gaussian MIMO BC-MAC Duality With Multiple Transmit Covariance Constraints ${ }^{\star}$
}

\author{
Lan Zhang $^{\dagger}$, Rui Zhang ${ }^{\ddagger}$, Ying-Chang Liang ${ }^{\ddagger}$, Yan $\mathrm{Xin}^{\dagger \dagger}$, and H. Vincent Poor*
}

\section{Suggested Editorial Areas:}

Communications, Shannon Theory

* This work was supported by the National University of Singapore (NUS) under start-up grants R-263-000-314-101, R-263-000-314112, and R-263-000-478-112, by a NUS Research Scholarship, and by the U.S. National Science Foundation under Grants ANI-03-38807 and CNS-06-25637. This work was partially done while Y. Xin was visiting Princeton University.

${ }^{\dagger}$ L. Zhang is with the Department of Electrical and Computer Engineering, National University of Singapore, Singapore 118622 (email: zhanglan@nus.edu.sg).

${ }^{\ddagger}$ R. Zhang and Y.-C. Liang are with Institute of Infocomm Research, A*STAR, 21 Heng Mui Keng Terrace, Singapore 119613 (email: rzhang@i2r.a-star.edu.sg; ycliang@i2r.a-star.edu.sg).

${ }^{\dagger \dagger}$ Y. Xin is with the NEC Laboratories America, Princeton, NJ 08540, USA (email: yanxin@nec-labs.com).

${ }^{*}$ H. V. Poor is with the Department of Electrical Engineering, Princeton University, Princeton, NJ 08544, USA (email: poor@princeton.edu). 


\begin{abstract}
Owing to the structure of the Gaussian multiple-input multiple-output (MIMO) broadcast channel (BC), associated optimization problems such as capacity region computation and beamforming optimization are typically non-convex, and cannot be solved directly. One feasible approach to these problems is to transform them into their dual multiple access channel (MAC) problems, which are easier to deal with due to their convexity properties. The conventional BC-MAC duality is established via BC-MAC signal transformation, and has been successfully applied to solve beamforming optimization, signal-to-interference-plus-noise ratio (SINR) balancing, and capacity region computation. However, this conventional duality approach is applicable only to the case, in which the base station (BS) of the BC is subject to a single sum power constraint. An alternative approach is minimax duality, established by $\mathrm{Yu}$ in the framework of Lagrange duality, which can be applied to solve the per-antenna power constraint problem. This paper extends the conventional BC-MAC duality to the general linear constraint case, and thereby establishes a general BC-MAC duality. This new duality is applied to solve the capacity computation and beamforming optimization for the MIMO and multiple-input single-output (MISO) BC, respectively, with multiple linear constraints. Moreover, the relationship between this new general BC-MAC duality and minimax duality is also presented. It is shown that the general BC-MAC duality offers more flexibility in solving BC optimization problems relative to minimax duality. Numerical results are provided to illustrate the effectiveness of the proposed algorithms.
\end{abstract}

\title{
I. INTRODUCTION
}

In a Gaussian multiple input multiple output (MIMO) broadcast channel (BC), the base station (BS) equipped with multiple transmit antennas sends independent information to each of multiple remote users, which are equipped with multiple receive antennas. In the past decade, a great deal of research has been focused on the characterization of optimal transmission schemes for the MIMO BC [1]-[6]. Due to the coupled structure of the transmitted signals, the optimization problems associated with the $\mathrm{BC}$ are usually non-convex. The key technique used to overcome this difficulty is to transform the BC problem into a convex multiple access channel (MAC) problem via a so-called BC-MAC duality relationship. Up to now, two types of $\mathrm{BC}-\mathrm{MAC}$ duality have been proposed as follows:

1) Conventional BC-MAC Duality ( [1], [3], [7], [8]):

Under a single sum power constraint, the capacity region (or signal-to-interference-plus-noise- 
ratio (SINR) region) of a $\mathrm{BC}$ is identical to that of a dual MAC under the same sum power constraint. The channel matrix associated with the dual MAC is the conjugate transposed channel matrix of the $\mathrm{BC}$, and the noise covariance matrices of both channels are identity matrices [1], [9].

2) Minimax Duality ( [5], [10], [11]):

The sum rate maximization problem of a $\mathrm{BC}$ with multiple linear constraints has the same solution as the dual MAC minimax optimization problem. The channel matrix of the dual MAC is the conjugate transposed channel matrix of the $\mathrm{BC}$, and the noise covariance matrix of the dual MAC is an unknown variable of the minimax optimization problem [10].

The conventional BC-MAC duality was first observed by Rashid-Rarrokhi et al. [1], and applied to solve the sum power minimization problem for a BC with SINR constraints. Several different methods have been developed independently to prove the conventional BC-MAC duality. The proof in [1] is based on the equivalent transformation that maps the SINR of the MAC to that of the BC. Vishwanath et al. [12] proved the conventional BC-MAC duality by presenting the explicit transformation between the transmit covariance matrix of the $\mathrm{BC}$ and that of the $\mathrm{MAC}$, and applied this duality to solve the sum capacity problem. Both proofs here are based on the reciprocity relation [13] between the BC and its dual MAC. Another proof based on the Karash-Kuhn-Tucker (KKT) conditions was given by Visotsky and Madhow [14]. The conventional BC-MAC duality has been widely applied to many BC problems. Schubert and Boche [15] solved an BC SINR balancing problem, which is to maximize the minimal SINR among all the users under a sum power constraint, via transforming the problem into its dual MAC problem. The conventional BC-MAC duality was also used to show that a dirty paper coding (DPC) [16] is a sum-capacity achieving strategy by Viswanath and Tse [8]. Moreover, the entire capacity region for the MIMO BC channel can be obtained via the conventional BC-MAC duality [3], [4], [17]. However, the conventional BC-MAC duality is applicable only to the case in which the BS of the $\mathrm{BC}$ is subject to a single sum power constraint.

On the other hand, the sum-capacity for the MIMO-BC was also studied by Yu and Cioffi [11] via minimax optimization. The minimax duality was proposed by $\mathrm{Yu}[10]$, where the conventional BCMAC duality and the minimax duality are unified in the framework of Lagrange duality. However, only 
the sum capacity is considered in [10]. Furthermore, Yu and Lan [5] extended the minimax duality to solve the capacity region computation problem and beamforming problem for the $\mathrm{BC}$ with per-antenna power constraint. The proofs of the minimax duality in [5] and [10] are based on Lagrange duality. Compared with the conventional BC-MAC duality, the minimax duality can be applied to the case with multiple linear constraints. However, since the dual MAC problem has a minimax form, and the noise covariance matrix of the dual MAC is unknown, high-efficiency algorithms, such as the iterative water-filling algorithm [18], cannot be applied.

\section{A. Overview of the Main Results}

The purpose of this paper is to establish the general BC-MAC duality via the BC-MAC SINR transformation, and unify the BC-MAC duality and the minimax duality in the framework of the reciprocity relationship between the $\mathrm{BC}$ and the MAC. By introducing several auxiliary variables and applying the general BC-MAC duality, the primal BC problem with multiple transmit covariance constraints is transformed into its dual MAC problem with a single sum power constraint and can

be efficiently solved via the existing algorithm for its dual MAC as the MAC problem has a convex structure that is easier to handle.

In this paper, we first consider a MIMO BC with a single general linear constraint. Relying on the $\mathrm{BC}-\mathrm{MAC}$ transformation, we prove that the capacity region of the $\mathrm{BC}$ is the same as that of its dual MAC with a single weighted sum power constraint, which we term the general $B C$-MAC duality. The channel matrix of the dual MAC is the transposed channel matrix of the primal $\mathrm{BC}$, and its noise covariance matrix is the coefficient of the linear constraint instead of being an identity matrix as in the conventional BC-MAC duality.

To exploit the general BC-MAC duality, the weighted sum rate maximization problem for the $\mathrm{BC}$ with multiple linear constraints is transformed into a single linear constraint problem by introducing several auxiliary variables. Though the rate maximization problem for the $\mathrm{BC}$ is a non-convex problem, we show that the KKT conditions are sufficient for optimality, and show that the subgradient-based algorithm converges to the optimal solution. A method for obtaining the subgradient is also given. The relationship between the general BC-MAC duality based solution and the minimax duality based solution [5] is explored. We show that the new method to handle multiple-constraint is equivalent to 
that of the minimax duality. But since the general BC-MAC duality based method solves the multiple constraint optimization problem in a decoupled manner, the new result has more flexibility to apply the existing algorithms for the MAC, while the minimax duality does not. Moreover, we discuss the weighted sum rate maximization problem with a convex but nonlinear constraint, and develop a new iterative algorithm to solve this optimization problem. In addition to the weighted sum rate maximization problem, the proposed method is also applied to solve the beamforming problem in a multiple-input single-output (MISO) BC with multiple linear constraints.

\section{B. Organization and Notation}

The remainder of this paper is organized as follows. The system model is described in Section II. Section III presents the general BC-MAC duality, where the transmit covariance matrix of the BC is subject to a linear constraint. The capacity region computation problem of the $\mathrm{BC}$ with multiple constraints or a single nonlinear constraint is studied in Section IV. The method to cope with multiple linear constraints is also applied to solve the beamforming problems in Section V. Several numerical results are provided in Section VI to illustrate the effectiveness of the proposed methods. Finally, Section VII concludes the paper.

Throughout this paper, we use boldface upper and boldface lower case letters for matrices and vectors, respectively. $(\cdot)^{H}$ denotes the matrix conjugate transpose operation, and $\operatorname{tr}(\cdot)$ denotes the matrix trace operation. $\mathbb{E}(\cdot)$ denotes the expectation operation for random variables. $I$ denotes an identity matrix.

\section{SySTEM MODEL}

We consider a MIMO BC system shown in Fig. 1 (a), where the BS intends to send independent information streams to each of $K$ remote users. The BS has $N_{t}$ transmit antennas and each user has $N_{r}$ receive antennas. The signal received by the $i$ th user is modeled as follows:

$$
\boldsymbol{y}_{i}=\boldsymbol{H}_{i} \boldsymbol{x}+\boldsymbol{z}_{i}, i=1, \cdots, K
$$

where the $N_{t} \times 1$ vector $\boldsymbol{x}$ denotes the transmit signal at the BS, $\boldsymbol{H}_{i}$ denotes the $N_{r} \times N_{t}$ channel matrix from the BS to the $i$ th user, $\boldsymbol{y}_{i}$ denotes the receive signal at the $i$ th user, and $\boldsymbol{z}_{i}$ is the noise vector. The entries of $\boldsymbol{z}_{i}$ are modeled as independent and identically distributed complex Gaussian random 
variables with mean zero and variance $\sigma_{i}^{2}$. The transmit signal covariance matrix of the BS is defined as $\boldsymbol{Q}:=\mathbb{E}\left(\boldsymbol{x} \boldsymbol{x}^{H}\right)$. In this paper, we assume that the channel knowledge is perfectly known at both the BS and the users, i.e., $\boldsymbol{H}_{i}$ is perfectly known at the transmitter and the receivers.

Since the transmit signals for different users are independent, we have

$$
\boldsymbol{x}=\sum_{i=1}^{K} \boldsymbol{x}_{i}
$$

where $\boldsymbol{x}_{i}$ denotes the transmit signal intended for the $i$ th user. Furthermore, to fully utilize the spatial diversity of the multi-antenna system, a spatial multiplexing scheme is also applied, which means that the data intended for each user is further divided into $N$ substreams, where $N=\min \left(N_{t}, N_{r}\right)$ [13]. Thus, the transmit signal for the $i$ th user can be expressed as follows:

$$
\boldsymbol{x}_{i}=\sum_{j=1}^{N} \boldsymbol{u}_{i, j} b_{i, j}
$$

where $b_{i, j}$ is a complex scalar variable with $p_{i, j}:=\mathbb{E}\left(\left|b_{i, j}\right|^{2}\right)$, representing the information signal of the $j$ th data substream of the $i$ th user, and $\boldsymbol{u}_{i, j}$ denotes the corresponding beamforming vector with $\left\|\boldsymbol{u}_{i, j}\right\|=1$. Combining (2), (3) and the definition of the transmit covariance matrix $\boldsymbol{Q}$, we have

$$
\boldsymbol{Q}=\sum_{i=1}^{K} \sum_{j=1}^{N} p_{i, j} \boldsymbol{u}_{i, j} \boldsymbol{u}_{i, j}^{H}
$$

\section{A. Nonlinear Encoding and Decoding Strategy}

It has been shown that the DPC scheme is a capacity achieving scheme for the Gaussian MIMO $\mathrm{BC}$ [17]. With the DPC scheme, the information for different users is encoded in a sequential manner. Without loss of generality, we assume that the encoding order is identical to the index order, i.e., the data substream $b_{1,1}$ is encoded first, $b_{1,2}$ is next encoded, and so on. According to the DPC scheme, the latter encoded data stream has non-causal information about its former encoded data streams, and thus the interference caused by the former data streams' transmission can be completely removed by the DPC scheme. Thus, the rate achieved by the $i$ th user can be expressed as

$$
r_{i}=\log \frac{\left|\sigma_{i}^{2} \boldsymbol{I}+\boldsymbol{H}_{i}\left(\sum_{k=i}^{K} \boldsymbol{Q}_{i}\right) \boldsymbol{H}_{i}^{H}\right|}{\left|\sigma_{i}^{2} \boldsymbol{I}+\boldsymbol{H}_{i}\left(\sum_{k=i+1}^{K} \boldsymbol{Q}_{i}\right) \boldsymbol{H}_{i}^{H}\right|}
$$

where $\boldsymbol{Q}_{i}:=\mathbb{E}\left(\boldsymbol{x}_{i} \boldsymbol{x}_{i}^{H}\right)$. 


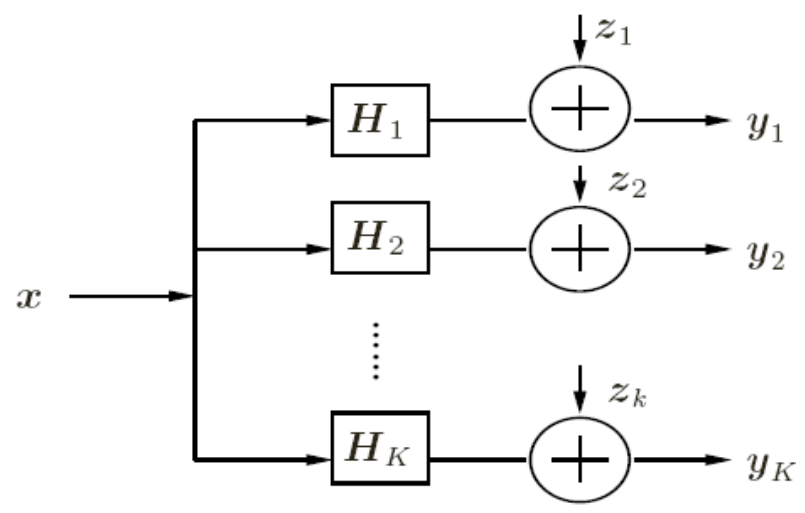

(a) BC, $\boldsymbol{z}_{i} \sim \mathcal{N}\left(0, \sigma_{i}^{2} \boldsymbol{I}\right), \boldsymbol{Q} \boldsymbol{A} \leq P$

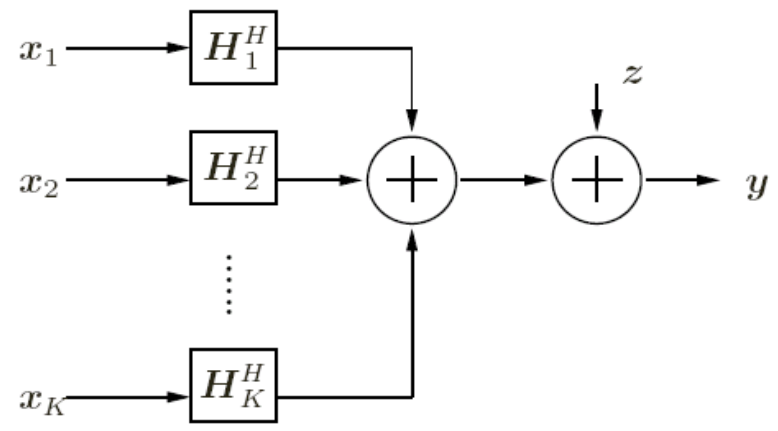

(b) Dual MAC, $\boldsymbol{z} \sim \mathcal{N}(0, \boldsymbol{A}), \sum_{i=1}^{K} \sigma_{i}^{2} \operatorname{tr}\left(\boldsymbol{Q}_{i}^{(\mathrm{m})}\right) \leq P$

Fig. 1. The system models for the primal MIMO BC and the dual MAC.

At the receiver side, for each user, successive interference cancellation (SIC) and the linear minimum mean square error (MMSE) filter are adopted to decode the corresponding information. With SIC, the first data stream is decoded by treating all the other streams as interference; then the signal from the first data stream is subtracted from the received signal, and the second data stream is decoded next, and so on. Thus, the mutual information between the BS and the $i$ th user can be expressed as

$$
I\left(\boldsymbol{x}_{i} ; \boldsymbol{y}_{i}\right)=I\left(b_{i, 1}, \cdots, b_{i, N} ; \boldsymbol{y}_{i}\right)=I\left(b_{i, 1} ; \boldsymbol{y}_{i}\right)+I\left(b_{i, 2} ; \boldsymbol{y}_{i} \mid b_{i, 1}\right)+\cdots+I\left(b_{i, N} ; \boldsymbol{y}_{i} \mid b_{i, 1}, \cdots, b_{i, N-1}\right) .
$$

Moreover, since the MMSE receiver is information-lossless [19], each term in (6) is achievable with the MMSE receiver. Thus, the MMSE-SIC receiver can achieve the capacity of the MIMO system. The receive beamforming vector for the $j$ th data substream at the $i$ th user is denoted by the $N_{r} \times 1$ vector $\boldsymbol{v}_{i, j}$. Thus, the SINR of the $j$ th data substream at the $i$ th user receiver can be written as

$$
\operatorname{SINR}_{i, j}=\frac{p_{i, j}\left|\boldsymbol{v}_{i, j}^{H} \boldsymbol{H}_{i} \boldsymbol{u}_{i, j}\right|^{2}}{\sum_{k=i+1}^{K} \sum_{l=1}^{N} p_{k, l}\left|\boldsymbol{v}_{i, j}^{H} \boldsymbol{H}_{i} \boldsymbol{u}_{k, l}\right|^{2}+\sum_{l=j+1}^{N} p_{i, l}\left|\boldsymbol{v}_{i, j}^{H} \boldsymbol{H}_{i} \boldsymbol{u}_{i, l}\right|^{2}+\sigma_{i}^{2}} .
$$

Since the achievable rate of each data substream depends on its SINR, we have

$$
r_{i}=\sum_{j=1}^{N} \log \left(1+\operatorname{SINR}_{i, j}\right)
$$




\section{B. Linear Encoding and Decoding Strategy}

Although the nonlinear strategies DPC and SIC are capacity-achieving schemes, they are difficult to implement in practice. A straightforward scheme for transmission is beamforming without DPC at the transmitter side and SIC at the receiver side. In the linear strategy, the transmit and receive beamforming vectors for the $j$ th data substream of the $i$ th user are still denoted by $\boldsymbol{u}_{i, j}$ and $\boldsymbol{v}_{i, j}$, respectively. Thus, the corresponding SINR can be expressed as

$$
\operatorname{SINR}_{i, j}^{(1)}=\frac{p_{i, j}\left|\boldsymbol{v}_{i, j}^{H} \boldsymbol{H}_{i} \boldsymbol{u}_{i, j}\right|^{2}}{\sum_{k=1}^{K} \sum_{l=1}^{N}(k, l) \neq(i, j) p_{k, l}\left|\boldsymbol{v}_{i, j}^{H} \boldsymbol{H}_{i} \boldsymbol{u}_{k, l}\right|^{2}+\sigma_{i}^{2}} .
$$

Since the method developed in the present paper is applicable to both linear and nonlinear strategies, we mainly focus on the nonlinear strategy, and adopt the SINR definition (7) throughout the paper.

\section{General Linear Transmit Covariance Constraint}

In the aforementioned literature, the transmit covariance matrix is subject only to a sum power constraint or/and a per-antenna power constraint. In this paper, we consider a general linear transmit covariance constraint as follows:

$$
\operatorname{tr}(\boldsymbol{Q} \boldsymbol{A}) \leq P
$$

where $\boldsymbol{A}$ is an $N_{t} \times N_{t}$ matrix, and $P$ is a constant. If the matrix $\boldsymbol{A}$ is chosen to be an identity matrix, then the constraint (10) is reduced to the sum power constraint; if the matrix $\boldsymbol{A}$ is chosen to be the diagonal matrix having all diagonal elements being zero except the $j$ th element being 1 , then the constraint (10) is reduced to the $j$ th antenna power constraint. In cognitive radio networks, we choose $\boldsymbol{A}=\boldsymbol{h} \boldsymbol{h}^{H}$, where $\boldsymbol{h}$ is the channel response from the secondary transmitter to the primary receiver, and the constraint is reduced to the interference power constraint for protecting the primary users [20]-[23]. Therefore, the constraint (10) can be viewed as a generalized linear constraint.

\section{Objective Functions}

In this paper, we consider several scenarios with different objectives: MIMO BC capacity region computation, SINR balancing, and power balancing.

1) MIMO BC Capacity Region Computation 
Any boundary rate tuple of the Gaussian MIMO BC capacity region can be obtained by solving the weighted sum rate maximization problem with some given user rate weights. Therefore, the capacity region computation problem is formulated as follows:

$$
\max _{\boldsymbol{Q} \geq 0} \sum_{i=1}^{K} w_{i} r_{i}
$$

where $w_{i}$ is the positive weight of the $i$ th user, and $\boldsymbol{Q} \geq 0$ denotes the semidefiniteness constraint. By varying the values of the weight $w_{i}$ s, the entire capacity region of the MIMO BC can be obtained.

\section{2) SINR Balancing}

The aim of the SINR balancing problem is to maximize the minimal ratio between the achieved SINR and the target SINR among all the data substreams. Mathematically, the optimization problem is formulated as

$$
\max _{Q \geq 0} \min _{\forall i, j} \frac{\operatorname{SINR}_{i, j}}{\gamma_{i, j}}
$$

where $\gamma_{i, j}$ is the target SINR for the $j$ th data substream of the $i$ th user. Conventionally, the SINR balancing problem considers the MISO case [15], i.e., $N_{t}=1$. It has been shown in [15] that the ratios of all the data substreams are equal, when the optimal solution is achieved. Therefore, the problem is termed the SINR "balancing" problem. Note that (12) is equivalent to the following form

$$
\begin{gathered}
\max _{\boldsymbol{Q} \geq 0, \alpha} \alpha \\
\text { subject to } \operatorname{SINR}_{i, j} \geq \alpha \gamma_{i, j}, \forall i, j .
\end{gathered}
$$

In the sequel, we will use (13) as the optimization problem for the SINR balancing problem instead of (12), since it is easier to write the Lagrange function of (13).

\section{3) Power Balancing}

In this case, the system has several different power requirements, and the objective is to minimize the maximal ratio between the transmit power and the power requirement. Mathematically, the optimization problem can be expressed as

$$
\min _{\boldsymbol{Q} \geq 0} \max _{\forall i} \frac{\operatorname{tr}\left(\boldsymbol{Q} \boldsymbol{A}_{i}\right)}{P_{i}}
$$


where $P_{i}$ is the $i$ th power requirement, and $\boldsymbol{A}_{i}$ is an $N_{t} \times N_{t}$ matrix for the $i$ th power requirement. Similarly to the SINR balancing function, the problem (14) can be transformed into

$$
\begin{gathered}
\min _{\boldsymbol{Q} \geq 0, \alpha} \alpha \\
\text { subject to } \operatorname{tr}\left(\boldsymbol{Q} \boldsymbol{A}_{i}\right) \leq \alpha P_{i}, \forall i .
\end{gathered}
$$

If there is a single power requirement, and the corresponding matrix $\boldsymbol{A}$ is an identity matrix, then (14) reduces to the power minimization problem.

In this paper, we will consider these optimization problems with several general transmit covariance constraints or SINR constraints.

\section{GENERAL BC-MAC DUALITY}

In this section, we establish the general BC-MAC duality under a single linear transmit covariance constraint. We start with the SINR balancing problem expressed as follows:

$$
\begin{aligned}
& \max _{\boldsymbol{Q} \geq 0, \alpha} \alpha \\
& \text { subject to } \operatorname{SINR}_{i, j} \geq \alpha \gamma_{i, j}, \quad \forall i, j \\
& \operatorname{tr}(\boldsymbol{Q} \boldsymbol{A}) \leq P .
\end{aligned}
$$

The MIMO BC SINR balancing problem (16) is a non-convex optimization problem due to the nonconvex SINR constraints. Although it has been shown in [5], [24] that the SINR constraint under the MISO scenario can be transformed into the second order cone (SOC) form, the transformation is not applicable to the MIMO case due to the essentially non-convex property of the MIMO SINR constraints. Hence, the problem (16) is still an open problem, and cannot be solved via existing methods. However, we can establish a new MAC called the dual MAC, and formulate a dual MAC problem of the primal problem (16) such that it shares the same solution as its primal problem (16).

Definition 1: The dual MAC of the primal BC in (1) has the conjugate transposed channel matrix of the $\mathrm{BC}$, i.e., the channel matrix of the dual MAC from the $i$ th user to the $\mathrm{BS}$ is $\boldsymbol{H}_{i}^{H}$, and the noise covariance matrix at the BS is the matrix $\boldsymbol{A}$ instead of the identity matrix, which is shown in the Fig. 1 (b). 
The corresponding dual MAC optimization problem is expressed as follows:

$$
\begin{gathered}
\max _{\boldsymbol{Q}_{i}^{(m)} \geq 0, \alpha} \alpha \\
\text { subject to } \operatorname{SINR}_{i, j}^{(m)} \geq \alpha \gamma_{i, j}, \quad \forall i, j \\
\qquad \sum_{i=1}^{K} \sigma_{i}^{2} \operatorname{tr}\left(\boldsymbol{Q}_{i}^{(m)}\right) \leq P
\end{gathered}
$$

where $\boldsymbol{Q}_{i}^{(m)}$ denotes the transmit signal covariance matrix of the $i$ th user, $\operatorname{SINR}_{i, j}^{(m)}$ denotes the SINR of the $j$ th data substream of the $i$ th user, and the superscript ' $(m)$ ' indicates that the corresponding variables are for the dual MAC. In this dual MAC, the MMSE-SIC scheme is applied, which means that the data streams of the dual MAC are decoded in a sequential manner. In the dual MAC problem (17), the decoding order at the BS is converse to the encoding order of the DPC at the primal BC. Accordingly, the $\operatorname{SINR}_{i, j}^{(m)}$ can be expressed as

$$
\operatorname{SINR}_{i, j}^{(m)}=\frac{q_{i, j}\left|\boldsymbol{u}_{i, j} \boldsymbol{H}_{i}^{H} \boldsymbol{v}_{i, j}\right|^{2}}{\boldsymbol{u}_{i, j}^{H}\left(\sum_{k=1}^{i-1} \sum_{l=1}^{N} q_{k, l} \boldsymbol{H}_{k}^{H} \boldsymbol{v}_{k, l} \boldsymbol{v}_{k, l}^{H} \boldsymbol{H}_{k}+\sum_{l=1}^{j-1} q_{i, l} \boldsymbol{H}_{i}^{H} \boldsymbol{v}_{i, l} \boldsymbol{v}_{i, l}^{H} \boldsymbol{H}_{i}+\boldsymbol{A}\right) \boldsymbol{u}_{i, j}}
$$

where $q_{i, j}$ is the power allocated to this data substream, and $\boldsymbol{u}_{i, j}$ and $\boldsymbol{v}_{i, j}$ denote the corresponding receive and transmit beamforming vectors, respectively. While it may be somewhat confusing at first that the beamforming vectors of the dual MAC share the same notation with the beamforming vectors of the primal $\mathrm{BC}$ in (7), it will become clear in the following that the optimal beamforming vectors of the primal $\mathrm{BC}$ are identical to those of the dual MAC.

The relationship between the primal problem (16) and the dual MAC problem (17) is summarized in the following proposition.

Proposition 1: The optimal solutions of the primal problem (16) and the dual MAC problem (17) are the same.

Proof: First, we will prove by contradiction that the SINR constraints for problem (16) hold with equality when the optimal solutions are achieved. If $\operatorname{SINR}_{i, j}>\alpha^{*} \gamma_{i, j}$ with $\alpha^{*}$ being the optimal solution of problem (16), then we can reduce part of the power $p_{i, j}$ and distribute it to all the other data substreams, thereby increasing the objective value $\alpha$ without violating the constraints. This contradicts $\alpha^{*}$ being the optimal solution. A similar argument holds for the problem (17). 
We next prove that if $\bar{\alpha}$ is achievable for the problem (16), then it is also achievable for the problem (17). Assume that when $\bar{\alpha}$ is achieved, $\overline{\boldsymbol{u}}_{i, j}$ and $\overline{\boldsymbol{v}}_{i, j}$ are the corresponding beamforming vectors for transmitter and receiver, respectively, and $\bar{p}_{i, j}$ is the power allocated to the $j$ th data substream of the $i$ th user. For the dual MAC problem (17), we can choose $\overline{\boldsymbol{v}}_{i, j}$ to be the transmit beamforming vector for the user side, and $\overline{\boldsymbol{u}}_{i, j}$ to be the receive beamforming vector at the BS. The power allocated to the $j$ th data substream of the $i$ th user of the dual MAC is assumed to be $\bar{q}_{i, j}$, which can be obtained by setting $\operatorname{SINR}_{i, j}^{(m)}=\operatorname{SINR}_{i, j}=\bar{\alpha} \gamma_{i, j}$, i.e.,

$$
\begin{aligned}
\bar{\alpha} \gamma_{i, j} & =\frac{\bar{p}_{i, j}\left|\overline{\boldsymbol{v}}_{i, j}^{H} \boldsymbol{H}_{i} \overline{\boldsymbol{u}}_{i, j}\right|^{2}}{\sum_{k=i+1}^{K} \sum_{l=1}^{N} \bar{p}_{k, l}\left|\overline{\boldsymbol{v}}_{i, j}^{H} \boldsymbol{H}_{i} \overline{\boldsymbol{u}}_{k, l}\right|^{2}+\sum_{l=j+1}^{N} \bar{p}_{i, l}\left|\overline{\boldsymbol{v}}_{i, j}^{H} \boldsymbol{H}_{i} \overline{\boldsymbol{u}}_{i, l}\right|^{2}+\sigma_{i}^{2}} \\
& =\frac{\bar{q}_{i, j}\left|\overline{\boldsymbol{u}}_{i, j} \boldsymbol{H}_{i}^{H} \overline{\boldsymbol{v}}_{i, j}\right|^{2}}{\overline{\boldsymbol{u}}_{i, j}^{H}\left(\sum_{k=1}^{i-1} \sum_{l=1}^{N} \bar{q}_{k, l} \boldsymbol{H}_{k}^{H} \overline{\boldsymbol{v}}_{k, l} \overline{\boldsymbol{v}}_{k, l}^{H} \boldsymbol{H}_{k}+\sum_{l=1}^{j-1} \bar{q}_{i, l} \boldsymbol{H}_{i}^{H} \overline{\boldsymbol{v}}_{i, l} \overline{\boldsymbol{v}}_{i, l}^{H} \boldsymbol{H}_{i}+\boldsymbol{A}\right) \overline{\boldsymbol{u}}_{i, j}} .
\end{aligned}
$$

By rearranging (19), we can list all the equations related to the SINR as follows:

$$
\begin{gathered}
\bar{p}_{1,1}\left(\overline{\boldsymbol{u}}_{1,1}^{H} \boldsymbol{A} \overline{\boldsymbol{u}}_{1,1}\right)=\bar{q}_{1,1}\left(\sum_{k=2}^{K} \sum_{l=1}^{N} \bar{p}_{k, l}\left|\overline{\boldsymbol{v}}_{1,1}^{H} \boldsymbol{H}_{1} \overline{\boldsymbol{u}}_{k, l}\right|^{2}+\sum_{l=2}^{N} \bar{p}_{1, l}\left|\overline{\boldsymbol{v}}_{1,1}^{H} \boldsymbol{H}_{1} \overline{\boldsymbol{u}}_{1, l}\right|^{2}+\sigma_{1}^{2}\right) \\
\bar{p}_{1,2}\left(\overline{\boldsymbol{u}}_{1,2}^{H}\left(\bar{q}_{1, l} \boldsymbol{H}_{1}^{H} \overline{\boldsymbol{v}}_{1, l} \overline{\boldsymbol{v}}_{1, l}^{H} \boldsymbol{H}_{1}+\boldsymbol{A}\right) \overline{\boldsymbol{u}}_{1,2}\right)=\bar{q}_{1,2}\left(\sum_{k=2}^{K} \sum_{l=1}^{N} \bar{p}_{k, l}\left|\overline{\boldsymbol{v}}_{1,2}^{H} \boldsymbol{H}_{1} \overline{\boldsymbol{u}}_{k, l}\right|^{2}+\sum_{l=3}^{N} \bar{p}_{1, l}\left|\overline{\boldsymbol{v}}_{1,2}^{H} \boldsymbol{H}_{1} \overline{\boldsymbol{u}}_{1, l}\right|^{2}+\sigma_{1}^{2}\right) \\
\vdots \\
\vdots \\
\bar{p}_{K, N}\left(\overline{\boldsymbol{u}}_{K, N}^{H}\left(\sum_{k=1}^{K-1} \sum_{l=1}^{N} \bar{q}_{k, l} \boldsymbol{H}_{k}^{H} \overline{\boldsymbol{v}}_{k, l} \overline{\boldsymbol{v}}_{k, l}^{H} \boldsymbol{H}_{k}+\sum_{l=1}^{N-1} \bar{q}_{K, l} \boldsymbol{H}_{K}^{H} \overline{\boldsymbol{v}}_{K, l} \overline{\boldsymbol{v}}_{K, l}^{H} \boldsymbol{H}_{K}+\boldsymbol{A}\right) \overline{\boldsymbol{u}}_{K, N}\right)=\bar{q}_{K, N} \sigma_{K}^{2} .
\end{gathered}
$$

By adding the above equations together, we have

$$
\sum_{i=1}^{K} \sum_{j=1}^{N} \bar{p}_{i, j} \overline{\boldsymbol{u}}_{i, j}^{H} \boldsymbol{A} \overline{\boldsymbol{u}}_{i, j}=\sum_{i=1}^{K} \sum_{j=1}^{N} \sigma_{i}^{2} \bar{q}_{i, j} .
$$

From the power constraint $\operatorname{tr}(\boldsymbol{Q} \boldsymbol{A}) \leq P$ of the problem (16), we have $\sum_{i=1}^{K} \sum_{j=1}^{N} \bar{p}_{i, j} \overline{\boldsymbol{u}}_{i, j}^{H} \boldsymbol{A} \overline{\boldsymbol{u}}_{i, j} \leq P$. Combining this with (20), we have $\sum_{i=1}^{K} \sum_{j=1}^{N} \sigma_{i}^{2} \bar{q}_{i, j} \leq P$, which means that the weighted sum power constraint $\sum_{i=1}^{K} \sigma_{i}^{2} \operatorname{tr}\left(\boldsymbol{Q}_{i}^{(m)}\right) \leq P$ of the problem (17) is satisfied. Hence, $\bar{\alpha}$ is achievable for the problem (17).

Contrarily, we can prove that if $\tilde{\alpha}$ is achievable for the problem (17), then $\tilde{\alpha}$ is also achievable for the problem (16).

The proof of Proposition 1 follows. 
If we assume that the optimal solution for both problems (16) and (17) is $\alpha^{*}$, then it means that the point $\left\{\operatorname{SINR}_{i, j}=\alpha^{*} \gamma_{i, j}, \forall i, j\right\}$ is achievable for the primal BC and the dual MAC with corresponding constraints, respectively. Therefore, under the general linear constraint $\operatorname{tr}(\boldsymbol{Q} \boldsymbol{A}) \leq P$, the primal $\mathrm{BC}$ can achieve the same SINR region as the dual MAC, which is subject to a weighted sum power constraint. From an information theoretic perspective, according to (8), the rate point $\left\{\boldsymbol{r}=\left[r_{1}, \cdots, r_{K}\right]\right\}$ is achievable if the $\operatorname{SINR}$ point $\left\{\operatorname{SINR}_{i, j}, \forall i, j\right\}$ is achievable under corresponding constraints. Thus, we have the following corollary. A rigorous proof is provided in Appendix A.

Corollary 1: The capacity region of the primal $\mathrm{BC}$ under the constraint $\operatorname{tr}(\boldsymbol{Q} \boldsymbol{A}) \leq P$, is equal to the capacity region of its dual MAC with a single weighted sum power constraint $\sum_{i=1}^{K} \sigma_{i}^{2} \operatorname{tr}\left(\boldsymbol{Q}_{i}^{(m)}\right) \leq P$.

Remark 1: By setting $\boldsymbol{A}$ in (16) to be an identity matrix, and assuming that $\sigma_{i}^{2}=1$ for all users, the general linear power constraint becomes a sum power constraint, and the noise covariance at the BS of the dual MAC is reduced to an identity matrix. This is precisely the same as the conventional BC-MAC duality. Thus, the new BC-MAC duality can be viewed as a generalization of the conventional BC-MAC duality. The proof of the duality is based on the special BC-MAC reciprocity relationship, instead of the Lagrange duality used in [5], [10]. Note that since the SINR constraints in (16) is not convex, the Lagrange duality gap between (16) and (17) may not be zero. Therefore, Proposition 1 cannot be proved through the use of Lagrange duality. But for the capacity region problem, the objective function is concave in the signal covariance matrices and convex in the noise covariance matrices. Hence, the Lagrange duality gap is zero and the Lagrange duality can be applied for the proof of the BC-MAC capacity duality. From this perspective, the reciprocity relationship is more fundamental than the Lagrange duality for the BC-MAC duality.

Remark 2: For a given set of transmit covariance matrices of the dual MAC, we can obtain the corresponding transmit covariance matrix of the primal $\mathrm{BC}$ to achieve the same value of $\alpha$ by using the method giving in the proof of Proposition 1. The detailed MAC-BC covariance matrix transformation algorithm is provided in Table I. Similarly, the BC-MAC covariance matrix transformation algorithm is readily obtained. Furthermore, the proof of Corollary 1 presents a MAC-BC covariance matrix transformation such that the primal $\mathrm{BC}$ and its dual MAC achieve the same rate tuple. The detailed 
TABLE I

THE MAC-BC COVARIANCE TRANSFORMATION FOR SINR EQUIVALENCE.

MAC-BC covariance matrix transformation I

1. Apply eigenvalue decomposition to $\boldsymbol{Q}_{i}^{(m)}=\boldsymbol{V}_{i} \boldsymbol{\Lambda}_{i} \boldsymbol{V}_{i}$ with $\boldsymbol{v}_{i, j}$ being the $j$ th column of $\boldsymbol{V}_{i}$ and $q_{i, j}$ being the $j$ th diagonal element of $\boldsymbol{\Lambda}_{i}$,

2. For each data substream, apply the MMSE algorithm to compute the receive beamforming vector $\boldsymbol{u}_{i, j}$, i.e.,

$$
\boldsymbol{u}_{i, j}=\left(\sum_{k=1}^{i-1} \sum_{l=1}^{N} \bar{q}_{k, l} \boldsymbol{H}_{k}^{H} \overline{\boldsymbol{v}}_{k, l} \overline{\boldsymbol{v}}_{k, l}^{H} \boldsymbol{H}_{k}+\sum_{l=1}^{j-1} \bar{q}_{i, l} \boldsymbol{H}_{i}^{H} \overline{\boldsymbol{v}}_{i, l} \overline{\boldsymbol{v}}_{i, l}^{H} \boldsymbol{H}_{i}+\boldsymbol{A}\right)^{-1} \boldsymbol{H}_{i}^{H} \overline{\boldsymbol{v}}_{i, j} .
$$

3. According to (19), compute $\boldsymbol{p}_{i, j}, \forall i, j$,

4. $\boldsymbol{Q}_{i}=\sum_{j=1}^{N} p_{i, j} \boldsymbol{u}_{i, j} \boldsymbol{u}_{i, j}^{H}$.

TABLE II

THE MAC-BC COVARIANCE TRANSFORMATION ALGORITHM FOR CAPACITY EQUIVALENCE.

\begin{tabular}{l}
\hline MAC-BC covariance matrix transformation II \\
\hline 1. Define $\hat{\boldsymbol{H}}_{k}=\boldsymbol{H}_{k} \boldsymbol{A}^{-1 / 2}, \boldsymbol{M}_{k}=\left(\boldsymbol{I}+\sum_{j=i+1}^{K} \hat{\boldsymbol{H}}_{j}^{H} \boldsymbol{Q}_{j}^{(m)} \hat{\boldsymbol{H}}_{j}\right)$ and \\
$\qquad \boldsymbol{B}_{k}=\left(\boldsymbol{I}+\hat{\boldsymbol{H}}_{k}\left(\sum_{j=i+1}^{K} \boldsymbol{Q}_{j}\right) \hat{\boldsymbol{H}}_{k}^{H}\right)$ \\
2. for $k=1, \cdots, K$ \\
3. calculate the SVD decomposition of $\boldsymbol{M}_{k}^{-1 / 2} \hat{\boldsymbol{H}}_{k} \boldsymbol{B}_{k}^{-1 / 2}=\boldsymbol{R}_{k} \boldsymbol{D}_{k} \boldsymbol{L}_{k}$ \\
4. $\quad \boldsymbol{Q}_{k}=\boldsymbol{A}^{-1 / 2} \boldsymbol{M}_{k}^{-1 / 2} \boldsymbol{R}_{k} \boldsymbol{L}_{k}^{H} \boldsymbol{B}_{k}^{1 / 2} \boldsymbol{Q}_{k}^{(m)} \boldsymbol{B}_{k}^{1 / 2} \boldsymbol{L}_{k} \boldsymbol{R}_{k}^{H} \boldsymbol{M}_{k}^{-1 / 2}\left(\boldsymbol{A}^{-1 / 2}\right)^{H}$ \\
5. end for \\
\hline
\end{tabular}

algorithm is presented in Table II.

Moreover, although Proposition 1 is for the nonlinear scheme, it is also applicable to the linear transmission scheme.

Corollary 2: Under the linear transmit strategy, the achievable SINR region of the primal BC under the constraint $\operatorname{tr}(\boldsymbol{Q A}) \leq P$, is equal to the achievable SINR region of its dual MAC with a single weighted sum power constraint $\sum_{i=1}^{K} \sigma_{i}^{2} \operatorname{tr}\left(\boldsymbol{Q}_{i}^{(m)}\right) \leq P$.

In the following, we will show how to use this general BC-MAC duality to solve various BC optimization problems with multiple transmit covariance constraints. 


\section{CAPACity Computation}

In the preceding section, the duality between the MIMO BC and the dual MAC with a general linear constraint was presented. In this section, by exploiting this duality, we will compute the capacity region of the MIMO BC. According to the discussion in Section II-D, the capacity of the BC can be obtained by solving the weighted sum rate maximization problem. For simplicity, we assume in the sequel that $\sigma_{i}^{2}=1$ for all the users.

\section{A. Single Linear Transmit Covariance Constraint}

We first consider the weighted sum rate maximization problem for the $\mathrm{BC}$ with a single linear constraint, which is formulated as follows:

$$
\max _{\boldsymbol{Q} \geq 0} \sum_{i=1}^{K} w_{i} r_{i}
$$

subject to $\operatorname{tr}(\boldsymbol{Q} \boldsymbol{A}) \leq P$

where $\boldsymbol{A}$ is a constant matrix, and $P$ is a constant. The problem (21) is a non-convex problem, and thus cannot be solved directly. According to Corollary 1, the problem (21) is equivalent to its dual MAC problem as follows:

$$
\begin{gathered}
\max _{\boldsymbol{Q}_{i}^{(m)} \geq 0} \sum_{i=1}^{K} w_{i} r_{i}^{(m)} \\
\text { subject to } \sum_{i=1}^{K} \operatorname{tr}\left(\boldsymbol{Q}_{i}^{(m)}\right) \leq P
\end{gathered}
$$

where $r_{i}^{(m)}:=\log \frac{\left|\boldsymbol{A}+\sum_{k=1}^{i} \boldsymbol{H}_{k}^{H} \boldsymbol{Q}_{k}^{(m)} \boldsymbol{H}_{k}\right|}{\left|\boldsymbol{A}+\sum_{k=1}^{i-1} \boldsymbol{H}_{k}^{H} \boldsymbol{Q}_{k}^{(m)} \boldsymbol{H}_{k}\right|}$ denotes the achievable rate of the $i$ th user. By solving the problem (22) via the interior point algorithm [25], the optimal solution for the problem (21) can be obtained via a MAC-BC transmit covariance matrix transformation algorithm.

In the following, we present an important property of the problem (21), which will be used in the case with multiple transmit covariance constraints. We first list the KKT conditions of the problem (21) as follows:

$$
\begin{array}{r}
\frac{\partial \sum_{i=1}^{K} w_{i} r_{i}}{\partial \boldsymbol{Q}_{i}}=\lambda \boldsymbol{A}+\boldsymbol{\Psi}_{i}, \quad \forall i \\
\lambda(\operatorname{tr}(\boldsymbol{Q} \boldsymbol{A})-P)=0
\end{array}
$$


where $\lambda$ is the Lagrange multiplier, and $\Psi_{i}$ is the Lagrange multiplier associated with the constraint $\boldsymbol{Q}_{i} \geq 0$. In general, the KKT conditions are only necessary for a solution to be optimal for a nonconvex problem. However, for the problem (21), it is shown in the following proposition that the KKT conditions are also sufficient for optimality.

Proposition 2: The KKT conditions (23) are sufficient for a solution to be optimal for the problem (21).

Proof: According to the Corollary 1, the problem (21) is equivalent to its dual MAC problem (23). We now assume that $\tilde{\boldsymbol{Q}}$ satisfies the KKT conditions in (23) and achieves the weighted sum rate $\tilde{R}$. Then, by the BC-MAC transmit covariance matrix transformation, we can obtain a set of $\tilde{\boldsymbol{Q}}_{i}^{(m)} \mathrm{s}$ for the problem (22) to achieve the same $\tilde{R}$. We next assume that $\bar{Q}$ is an optimal solution of the problem (21) with the optimal weighted sum rate $\bar{R}$, where $\bar{R}>\tilde{R}$. Thus, we can obtain the optimal solution of the problem (22) $\overline{\boldsymbol{Q}}_{i}^{(m)}$ by MAC-BC transmit covariance matrix transformation.

It is well known that the objective function of (22) is a convex function. Hence, we have $\boldsymbol{Q}_{i}^{*}:=$ $\tilde{\boldsymbol{Q}}_{i}^{(m)}+t\left(\overline{\boldsymbol{Q}}_{i}^{(m)}-\tilde{\boldsymbol{Q}}_{i}^{(m)}\right)$, where $0<t<1$, is a better solution than $\tilde{\boldsymbol{Q}}_{i}^{(m)}$ for the problem (22). Through the MAC-BC transmit covariance matrix transformation, we transform the dual MAC solution $\boldsymbol{Q}_{i}^{*}$ into its corresponding $\mathrm{BC}$ solution $Q^{*}$. Since the MAC-BC transmit covariance matrix transformation is continuous, we can always find a $t$ such that $\left\|\tilde{\boldsymbol{Q}}-\boldsymbol{Q}^{*}\right\| \leq \epsilon$ for a given $\epsilon>0$. Therefore, $\tilde{\boldsymbol{Q}}$ is not the local optimal solution, which is contradicted with the KKT condition $\partial\left(\sum_{i=1}^{K} w_{i} r_{i}\right) / \partial \boldsymbol{Q}_{i}=\lambda \boldsymbol{A}+\boldsymbol{\Psi}_{i}$. The proof thus follows.

\section{B. Multiple Linear Transmit Covariance Constraints}

We now consider the weighted sum rate maximization problem with multiple linear constraints as follows:

$$
\begin{aligned}
& \max _{\boldsymbol{Q} \geq 0} \sum_{i=1}^{K} w_{i} r_{i} \\
& \text { subject to } \operatorname{tr}\left(\boldsymbol{Q} \boldsymbol{A}_{1}\right) \leq P_{1} \\
& \operatorname{tr}\left(\boldsymbol{Q} \boldsymbol{A}_{2}\right) \leq P_{2}
\end{aligned}
$$


where $\boldsymbol{A}_{i}, i=1,2$, is an $N_{t} \times N_{t}$ constant matrix, and $P_{i}, i=1,2$, is a constant. For convenience of description, we discuss only the case of two linear constraints, though our method can be easily extended to the case of an arbitrary number of linear constraints.

Since the objective function is non-concave in $Q$, the problem (24) is not convex, and thus cannot be solved directly. In [3], [4], the sum capacity of the MIMO BC with a single sum-power constraint was studied. Based on the conventional BC-MAC duality [12], the $\mathrm{BC}$ problem was transformed into its dual convex MAC problem with a single sum-power constraint. However, the problem (24) is with multiple constraints, and thus the conventional BC-MAC duality cannot be applied. To exploit the general BC-MAC duality, we can transform the problem (24) into the following problem with a single constraint:

$$
\begin{gathered}
g\left(\lambda_{1}, \lambda_{2}\right):=\max _{\boldsymbol{Q} \geq 0} \sum_{i=1}^{K} w_{i} r_{i} \\
\text { subject to } \lambda_{1} \operatorname{tr}\left(\boldsymbol{Q} \boldsymbol{A}_{1}\right)+\lambda_{2} \operatorname{tr}\left(\boldsymbol{Q} \boldsymbol{A}_{2}\right) \leq \lambda_{1} P_{1}+\lambda_{2} P_{2}
\end{gathered}
$$

where $\lambda_{1}$ and $\lambda_{2}$ can be viewed as auxiliary variables. The relationship between the problem (24) and the problem (25) can be summarized as follows.

Proposition 3: The optimal solution of the problem (25) is an upper bound on that of the problem (24).

Proof: If $\boldsymbol{Q}$ is feasible for the problem (24), then it is also feasible for the problem (25). Therefore, the feasible region of the problem (24) is a subset of that of the problem (25). The proof follows immediately.

Furthermore, we can prove that the upper bound is tight, i.e., the optimal solution of the problem (24) achieves the upper bound $g\left(\lambda_{1}, \lambda_{2}\right)$ for some $\lambda_{1}$ and $\lambda_{2}$. Thus, we have

Proposition 4: The optimal solution of the problem (24) is equal to that of the problem $\min _{\lambda_{1}, \lambda_{2}} g\left(\lambda_{1}, \lambda_{2}\right)$.

Proof: The KKT condition of the problem (24) can be listed as follows:

$$
\begin{aligned}
\frac{\partial \sum_{i=1}^{K} w_{i} r_{i}}{\partial \boldsymbol{Q}_{i}}= & \mu_{1} \boldsymbol{A}_{1}+\mu_{2} \boldsymbol{A}_{2}+\boldsymbol{\Omega}_{i}, \forall i \\
& \mu_{1}\left(\operatorname{tr}\left(\boldsymbol{Q} \boldsymbol{A}_{1}\right)-P_{1}\right)=0 \\
& \mu_{2}\left(\operatorname{tr}\left(\boldsymbol{Q} \boldsymbol{A}_{2}\right)-P_{2}\right)=0
\end{aligned}
$$


where $\mu_{1}$ and $\mu_{2}$ are the Lagrange multipliers with respect to the two constraints, respectively, and $\Omega_{i}$ is the Lagrange multiplier associated with the constraint $\boldsymbol{Q}_{i} \geq 0$. When the optimal solution of the problem (24) is achieved, we assume that the corresponding optimal variables are $Q^{*}, \mu_{1}^{*}, \mu_{2}^{*}$, and $\Omega_{i}^{*}$.

We now list the KKT conditions of the problem (25) as follows:

$$
\begin{array}{r}
\frac{\partial \sum_{i=1}^{K} w_{i} r_{i}}{\partial \boldsymbol{Q}_{i}}=\lambda\left(\lambda_{1} \boldsymbol{A}_{1}+\lambda_{2} \boldsymbol{A}_{2}\right)+\boldsymbol{\Upsilon}_{i}, \quad \forall i \\
\lambda\left(\lambda_{1} \operatorname{tr}\left(\boldsymbol{Q} \boldsymbol{A}_{1}\right)+\lambda_{2} \operatorname{tr}\left(\boldsymbol{Q} \boldsymbol{A}_{2}\right)-\mu_{1} P_{1}-\mu_{2} P_{2}\right)=0
\end{array}
$$

where $\lambda$ is the Lagrange multiplier, and $\Upsilon_{i}$ is the Lagrange multiplier associated with the constraint $\boldsymbol{Q}_{i} \geq 0$. If we choose $\boldsymbol{Q}=\boldsymbol{Q}^{*}, \lambda=1, \lambda_{1}=\mu_{1}^{*}, \lambda_{2}=\mu_{2}^{*}$, and $\Upsilon_{i}=\boldsymbol{\Omega}_{i}^{*}$, the KKT conditions (27) are satisfied. Since the problem (25) is the weighted sum rate maximization problem with a single linear constraint, according to Proposition 2, the solution is the optimal solution of the problem (25). Combining this with Proposition 3, the proof follows.

According to the general BC-MAC duality discussed in Section III, the problem (25) is equivalent to the following dual MAC problem:

$$
\begin{aligned}
\max _{\boldsymbol{Q}_{i}^{(m)} \geq 0} & \sum_{i=1}^{K} w_{i} r_{i}^{(m)} \\
\text { subject to } & \sum_{i=1}^{K} \operatorname{tr}\left(\boldsymbol{Q}_{i}^{(m)}\right) \leq \lambda_{1} P_{1}+\lambda_{2} P_{2}
\end{aligned}
$$

where the noise covariance at the BS of the dual MAC is $\lambda_{1} \boldsymbol{A}_{1}+\lambda_{2} \boldsymbol{A}_{2}$, and $r_{i}^{(m)}$ denotes the achievable rate of the $i$ th user. The problem (28) is a convex optimization problem that can be solved via a standard interior point algorithm. With the optimal solution of the problem (28), the optimal solution for the problem (25) can be obtained by the MAC-BC transmit covariance matrix transformation. We next consider the minimization problem

$$
\min _{\lambda_{1} \geq 0, \lambda_{2} \geq 0} g\left(\lambda_{1}, \lambda_{2}\right)
$$

Since the function $g\left(\lambda_{1}, \lambda_{2}\right)$ is not necessarily differentiable, we can solve the problem (29) via the subgradient algorithm or ellipsoid algorithm. The subgradient of the function $g\left(\lambda_{1}, \lambda_{2}\right)$ can be found in the following proposition. Refer to Appendix B for the proof. 
Proposition 5: The subgradient of the function $g\left(\lambda_{1}, \lambda_{2}\right)$ at point $\left[\bar{\lambda}_{1}, \bar{\lambda}_{2}\right]$ is $\left[P_{1}-\operatorname{tr}\left(\overline{\boldsymbol{Q}} \boldsymbol{A}_{1}\right), P_{2}-\right.$ $\left.\operatorname{tr}\left(\overline{\boldsymbol{Q}} \boldsymbol{A}_{2}\right)\right]$, where $\overline{\boldsymbol{Q}}$ is the optimal solution of the inner layer optimization problem (28) with $\lambda_{1}=\bar{\lambda}_{1}$ and $\lambda_{2}=\bar{\lambda}_{2}$.

Remark 3: The Lagrangian function of the problem (24) can be written as

$$
\sum_{i=1}^{K} w_{i} r_{i}-\mu_{1}\left(\operatorname{tr}\left(\boldsymbol{Q} \boldsymbol{A}_{1}\right)-P_{1}\right)-\mu_{2}\left(\operatorname{tr}\left(\boldsymbol{Q} \boldsymbol{A}_{2}\right)-P_{2}\right)
$$

while the Lagrangian function of the problem (25) can be written as:

$$
\sum_{i=1}^{K} w_{i} r_{i}-\lambda\left(\lambda_{1} \operatorname{tr}\left(\boldsymbol{Q} \boldsymbol{A}_{1}\right)+\lambda_{2} \operatorname{tr}\left(\boldsymbol{Q} \boldsymbol{A}_{2}\right)-\lambda_{1} P_{1}-\lambda_{2} P_{2}\right) .
$$

By observing (30) and (31), we can say that the two Lagrange functions are identical to each other if we choose $\mu_{1}=\lambda \lambda_{1}$ and $\mu_{2}=\lambda \lambda_{2}$. Thus, the auxiliary variables $\lambda_{1}$ and $\lambda_{2}$ can be viewed as the Lagrange dual variables.

Since the function $g\left(\lambda_{1}, \lambda_{2}\right)$ is a convex function, the subgradient-based algorithm is guaranteed to converge to its optimal solution [26]. According to Proposition 4, when the minimum of $g\left(\lambda_{1}, \lambda_{2}\right)$ is achieved, the optimal solution of the problem (25) is equal to that of the problem (24).

In summary, the problem (24) is solved through a two-loop algorithm. By exploiting the general BC-MAC duality, the inner loop searches the optimal solution of $g\left(\lambda_{1}, \lambda_{2}\right)$, while the outer loop solves the $g\left(\lambda_{1}, \lambda_{2}\right)$ minimization problem via a subgradient-based iterative algorithm. The convexity of the function $g\left(\lambda_{1}, \lambda_{2}\right)$ guarantees that the global optimal solution is achieved.

\section{Relationship to Minimax Duality}

The capacity region computation problem with a per-antenna power constraint considered in [5] is a special case of the problem (24). By choosing $\boldsymbol{A}$ to be a diagonal matrix with all its diagonal elements being zero except the $j$ th diagonal element being 1, the constraint $\operatorname{tr}(\boldsymbol{Q} \boldsymbol{A}) \leq P$ can be viewed as the power constraint for the $j$ th antenna of the BS. Different from the method discussed in Section IV-B, 
the dual MAC problem developed in [5] has a minimax form as follows:

$$
\begin{aligned}
\min _{\hat{\boldsymbol{Q}}} \max _{\boldsymbol{Q}_{i}^{(\mathrm{mac})} \geq 0} & \sum_{k=1}^{K} w_{k} r_{k}^{(\mathrm{mac})} \\
\text { subject to } & \sum_{i=1}^{K} \operatorname{tr}\left(\boldsymbol{Q}_{i}^{(\mathrm{mac})}\right) \leq \operatorname{tr}(\boldsymbol{\Phi}) \\
& \operatorname{tr}(\hat{\boldsymbol{Q}} \boldsymbol{\Phi}) \leq \operatorname{tr}(\boldsymbol{\Phi})
\end{aligned}
$$

where $\Phi$ is a diagonal matrix,

$$
r_{k}^{(\mathrm{mac})}:=\log \frac{\left|\sum_{i=1}^{k} \boldsymbol{H}_{i}^{H} \boldsymbol{Q}_{i}^{(\mathrm{mac})} \boldsymbol{H}_{i}+\hat{\boldsymbol{Q}}\right|}{\left|\sum_{i=1}^{k-1} \boldsymbol{H}_{i}^{H} \boldsymbol{Q}_{i}^{(\mathrm{mac})} \boldsymbol{H}_{i}+\hat{\boldsymbol{Q}}\right|}
$$

and the diagonal matrix $\hat{\boldsymbol{Q}}$ is the noise covariance matrix of the dual MAC. The $j$ th diagonal element of $\Phi$ is the power constraint for the $j$ th antenna of the BS. Since the noise covariance is also an unknown variable, the existing high-efficiency algorithm for the MAC problem cannot be applied. Instead, a new interior point method based algorithm is developed in [5] to solve (32).

The two constraints in (32) have some redundancy, and can be further simplified via the following two observations.

1) The noise covariance constraint $\operatorname{tr}(\hat{\boldsymbol{Q}} \Phi) \leq \operatorname{tr}(\boldsymbol{\Phi})$ holds with equality when the optimal solution is achieved.

2) Given any positive $\alpha$, if we replace the constraint in (32) with $\sum_{i=1}^{K} \operatorname{tr}\left(\boldsymbol{Q}_{i}^{(\mathrm{mac})}\right) \leq \alpha \operatorname{tr}(\boldsymbol{\Phi})$ and $\operatorname{tr}(\hat{\boldsymbol{Q}} \Phi) \leq \alpha \operatorname{tr}(\boldsymbol{\Phi})$, the optimal value of the problem (32) does not change.

The observation 1) can be shown by observing that if the constraint $\operatorname{tr}(\hat{Q} \Phi) \leq \operatorname{tr}(\boldsymbol{\Phi})$ is satisfied with inequality, then the minimization part of the problem (32) does not achieve the optimal solution. The observation 2) can be proved through the KKT conditions. If we assume that $\boldsymbol{Q}_{i}^{*}$ and $\hat{Q}^{*}$ are the optimal transmit covariance matrix and the noise covariance matrix of the problem (32), respectively, then it is easy to verify that $\alpha \boldsymbol{Q}_{i}^{*}$ and $\alpha \hat{\boldsymbol{Q}}^{*}$ satisfy the KKT conditions of the problem (32) after the constraints replacement, and the optimal value of the problem (32) does not change.

Based on these two observations, we can combine the two constraints in (32) into one constraint 
$\sum_{i=1}^{K} \operatorname{tr}\left(\boldsymbol{Q}_{i}^{(\mathrm{mac})}\right) \leq \operatorname{tr}(\hat{\boldsymbol{Q}} \Phi)$, and thus the problem (32) is equivalent to the following problem:

$$
\begin{aligned}
\min _{\hat{\boldsymbol{Q}} \boldsymbol{Q}_{i}^{(\mathrm{mac})} \geq 0} & \sum_{k=1}^{K} w_{k} r_{k}^{(\mathrm{mac})} \\
\text { subject to } & \sum_{i=1}^{K} \operatorname{tr}\left(\boldsymbol{Q}_{i}^{(\mathrm{mac})}\right) \leq \operatorname{tr}(\hat{\boldsymbol{Q}} \boldsymbol{\Phi}) .
\end{aligned}
$$

In our derivation in Section IV-B, we can formulate a similar minimax optimization problem of the dual MAC. Combining (25), (28) and (29), we have

$$
\begin{aligned}
& \min _{\lambda_{1}, \lambda_{2}} \max _{\boldsymbol{Q}_{i}^{(m)} \geq 0} \sum_{i=1}^{K} w_{i} r_{i}^{(m)} \\
& \text { subject to } \sum_{i=1}^{K} \operatorname{tr}\left(\boldsymbol{Q}_{i}^{(m)}\right) \leq \lambda_{1} P_{1}+\lambda_{2} P_{2}
\end{aligned}
$$

where the noise covariance matrix of the dual MAC is $\lambda_{1} A_{1}+\lambda_{2} A_{2}$. In the per-antenna power constraint scenario, the problem (34) becomes

$$
\begin{aligned}
& \min _{\boldsymbol{S}} \max _{\boldsymbol{Q}_{i}^{(m)} \geq 0} \sum_{i=1}^{K} w_{i} r_{i}^{(m)} \\
& \text { subject to } \sum_{i=1}^{K} \operatorname{tr}\left(\boldsymbol{Q}_{i}^{(m)}\right) \leq \operatorname{tr}(\boldsymbol{S} \boldsymbol{\Phi})
\end{aligned}
$$

where $\boldsymbol{S}=\operatorname{diag}\left(\lambda_{1}, \cdots, \lambda_{N_{t}}\right)$. The problem (35) is identical to the problem (33) by noting that $\boldsymbol{S}=\hat{\boldsymbol{Q}}$ and $\boldsymbol{Q}_{i}^{(m)}=\boldsymbol{Q}_{i}^{(\mathrm{mac})}$. Therefore, the problem (32) and the problem (35) are equivalent to each other.

Although the general BC-MAC duality in Section III and the minimax duality in [10] have substantially different formulation, the ways by which they handle the multiple linear constraints are equivalent essentially. The general BC-MAC duality based method divides the process into two steps: dual MAC problem solving, and multiple constraints handling; while the minimax duality combines the two steps together. Essentially, only the dual MAC problem solving step exploits the special BC-MAC reciprocity relationship, and the multiple constraints handling step is not specific for the $\mathrm{BC}$ problem, i.e., it can be applied to solve any optimization problem with multiple constraints. The new method decouples the two steps, and thus has more flexibility in exploiting the existing algorithm for a MAC. Moreover, the minimax problem (32) is specifically formulated to solve the per-antenna power constraint problem. 


\section{Nonlinear Constraint}

According to the proof of Proposition 1, the general BC-MAC duality requires that the transmit covariance matrix of the $\mathrm{BC}$ be subject to a linear constraint. In this subsection, we consider the case with a convex but nonlinear constraint. The capacity computation under a nonlinear constraint can be formulated as follows:

$$
\begin{aligned}
\max _{\boldsymbol{Q} \geq 0} & \sum_{i=1}^{K} w_{i} r_{i} \\
\text { subject to } & f(\boldsymbol{Q}) \leq 0
\end{aligned}
$$

where the function $f(\boldsymbol{Q})$ is a general nonlinear convex function. Since the general BC-MAC duality does not hold generally under the nonlinear constraint, the problem (36) cannot be solved directly. However, as shown in the following proposition, the nonlinear constraint problem (36) is equivalent to a single linear constraint problem (refer to Appendix $\mathrm{C}$ for the proof).

Proposition 6: There always exists a linear constraint problem as follows

$$
\max _{\boldsymbol{Q} \geq 0} \sum_{i=1}^{K} w_{i} r_{i}
$$

$$
\text { subject to } \operatorname{tr}\left(\boldsymbol{A} \boldsymbol{Q}_{i}\right) \leq 0
$$

with $\boldsymbol{A}$ denoting a constant matrix such that it has the same solution as the original problem (36).

Remark 4: Proposition 6 illustrates that the nonlinear non-convex optimization problem (36) can be transformed into an equivalent linear constraint problem (37), which can be solved by making use of the general BC-MAC duality in Section III. However, according to the proof of Proposition 6, the parameter $\boldsymbol{A}$ in problem (37) cannot be obtained without the optimal solution $\boldsymbol{Q}^{*}$ of the problem (36). Note that it is impossible to obtain $Q^{*}$ before solving this problem, and thus the problem (36) cannot be solved by using its equivalence with the problem (37). In the following, we present an example to illustrate an iterative algorithm that can find a set of linear constraints, and these linear constraints can be used to approximate the original nonlinear constraint.

Example 1: For simplicity, we consider the MIMO BC with $K=2, N_{t}=2$, and $N_{r}=2$. The transmit covariance matrix is subject to a nonlinear constraint: $\left(\operatorname{tr}\left(\boldsymbol{Q} \boldsymbol{A}_{1}\right)\right)^{2}+\left(\operatorname{tr}\left(\boldsymbol{Q} \boldsymbol{A}_{2}\right)\right)^{2} \leq P$, where 
$\boldsymbol{A}_{1}=\left[\begin{array}{ll}1 & 0 \\ 0 & 0\end{array}\right]$, and $\boldsymbol{A}_{2}=\left[\begin{array}{cc}0 & 0 \\ 0 & 1\end{array}\right]$. Thus, the capacity computation under the nonlinear constraint can be formulated as follows:

$$
\max _{Q \geq 0} \sum_{i=1}^{2} w_{i} r_{i}
$$

$$
\text { subject to }\left(\operatorname{tr}\left(\boldsymbol{Q} \boldsymbol{A}_{1}\right)\right)^{2}+\left(\operatorname{tr}\left(\boldsymbol{Q} \boldsymbol{A}_{2}\right)\right)^{2} \leq P \text {. }
$$

As shown in Fig. 2, by defining $p_{1}:=\operatorname{tr}\left(\boldsymbol{Q} \boldsymbol{A}_{1}\right)$ and $p_{2}:=\operatorname{tr}\left(\boldsymbol{Q} \boldsymbol{A}_{2}\right)$, the feasible region $\mathbf{R}:\left\{p_{1}, p_{2} \mid p_{1}^{2}+\right.$ $\left.p_{2}^{2} \leq P, p_{1} \geq 0, p_{2} \geq 0\right\}$ is a quarter circle in the nonnegative orthant. According to Proposition 6 , the problem (38) is equivalent to the following problem

$$
\max _{Q \geq 0} \sum_{i=1}^{2} w_{i} r_{i}
$$

subject to $c_{1} \operatorname{tr}\left(\boldsymbol{Q} \boldsymbol{A}_{1}\right)+c_{2} \operatorname{tr}\left(\boldsymbol{Q} \boldsymbol{A}_{2}\right) \leq c_{3}$

where $c_{i}=\operatorname{tr}\left(\boldsymbol{Q}^{*} \boldsymbol{A}_{i}\right), i=1,2, \boldsymbol{Q}^{*}$ is the optimal solution of the problem (38), and $c_{3}=P$.

To present the iterative process, we use a graphical illustration as shown in Fig. 2. Firstly, we arbitrarily select a point 'a' on the boundary of the nonlinear region $\mathbf{R}$, and draw a tangent line to the $p_{1}^{2}+p_{2}^{2}=P$ curve through the selected point. The tangent line corresponds to a linear constraint $\mathbf{C}^{(1)}: c_{1}^{(1)} \operatorname{tr}\left(\boldsymbol{Q} \boldsymbol{A}_{1}\right)+c_{2}^{(1)} \operatorname{tr}\left(\boldsymbol{Q} \boldsymbol{A}_{2}\right)=c_{3}^{(1)}$, where the superscript '1' denotes the index of the linear constraints. The weighted sum rate maximization problem with the constraint $\mathbf{C}^{(1)}$ can be solved through the general duality. Assume that $\boldsymbol{Q}^{(1)}$ and $r^{(1)}$ are the corresponding optimal transmit covariance matrix and the obtained optimal weighted sum rate, respectively. Since the feasible region of the problem (38) is a subset of the feasible region of $\mathbf{C}^{(1)}, r^{(1)}$ is an upper bound on that of the original problem (38). The optimal transmit covariance matrix $\boldsymbol{Q}^{(1)}$, corresponding to the point $b$ in Fig. 2, is not feasible for the original problem. Secondly, we find the point 'c' on the boundary of the region $\mathbf{R}$ to be closest to the point ' $b$ ', and draw a new tangent line through the point ' $c$ '. The new tangent line corresponds to a linear constraint $\mathbf{C}^{(2)}: c_{1}^{(2)} \operatorname{tr}\left(\boldsymbol{Q} \boldsymbol{A}_{1}\right)+c_{2}^{(2)} \operatorname{tr}\left(\boldsymbol{Q} \boldsymbol{A}_{2}\right)=c_{3}^{(2)}$. By solving the weighted sum rate maximization problem with two constraints $\mathbf{C}^{(1)}$ and $\mathbf{C}^{(2)}$, a new optimal weighted sum rate $r^{(2)}$ is obtained, where $r^{(1)}>r^{(2)}$. The iterative process will continue until $\left(\operatorname{tr}\left(\boldsymbol{Q}^{(n)} \boldsymbol{A}_{1}\right)\right)^{2}+\left(\operatorname{tr}\left(\boldsymbol{Q}^{(n)} \boldsymbol{A}_{2}\right)\right)^{2} \leq P+\epsilon$ holds, 
where $Q^{(n)}$ denotes the optimal solution of the $n$th iterative step, and $\epsilon$ denotes the prescribed accuracy requirement. The obtained $r^{(n)}$ forms a non-increasing sequence with the lower bound on the optimal solution of the problem (38). Thus, the algorithm will converge to the optimal solution.
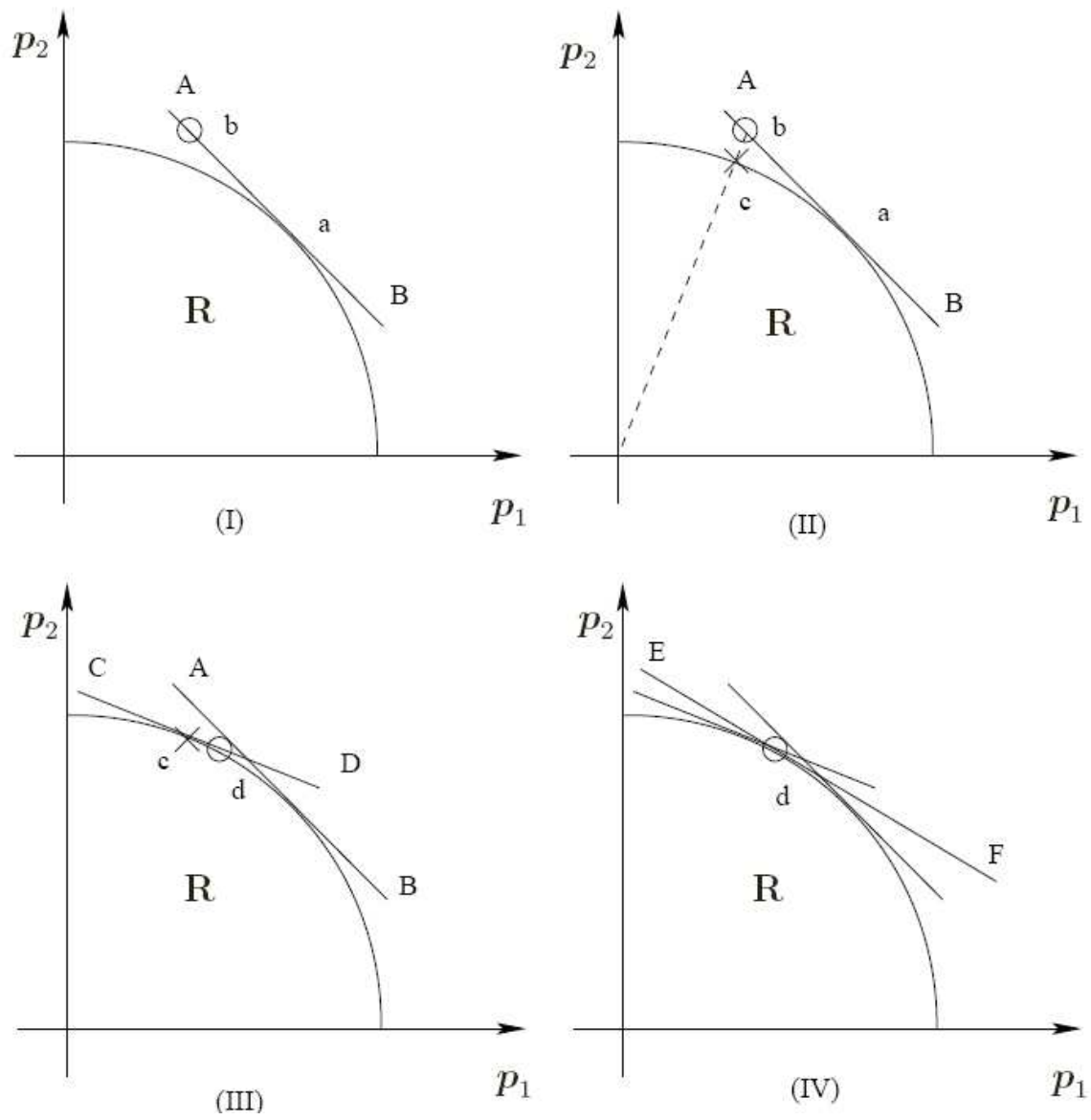

Fig. 2. The iterative process for the nonlinear constraint problem. (I) Select an initial point 'a', plot a tangent line 'AB', and solve the optimization problem with a single linear constraint with respect to 'AB', where point ' $\mathrm{b}$ ' corresponds to the optimal solution. (II) Find a point 'c', which is closest to 'b', on the boundary of the feasible region. (III) Plot a tangent line 'CD', and solve the optimization problem with the constraints 'AB' and 'CD', where 'd' is assumed to be the optimal solution. (IV) So on and so forth, until the optimal solution is achieved. 


\section{BEAMFORMing PROBLEM}

In the preceding section, the capacity computation for the MIMO BC is considered. In this section, we consider the MISO BC from a beamforming perspective ${ }^{1}$. Depending on the objective function and the constraints, the beamforming optimization problems can be divided into two classes. One is the SINR balancing problem [15], [27], i.e., maximizing the minimum SINR among all the users. The other one is the power minimization problem with SINR constraints [5], [24], [28], i.e., minimizing some power function with SINR constraints. In the case of $N_{r}=1$, the SINR of the $i$ th user under the nonlinear encoding and decoding scheme can be expressed as follows:

$$
\operatorname{SINR}_{i}=\frac{\left|\boldsymbol{h}_{i}^{H} \boldsymbol{u}_{i}\right|}{\sum_{j=i+1}^{K}\left|\boldsymbol{h}_{i}^{H} \boldsymbol{u}_{k}\right|^{2}+1}
$$

where $\boldsymbol{h}_{i}$ denotes the $N_{t} \times 1$ channel vector from the BS to the $i$ th user, and $\boldsymbol{u}_{i}$ denotes the transmit beamforming vector. Under the linear encoding and decoding scheme, the corresponding SINR can be expressed as follows:

$$
\operatorname{SINR}_{i}^{(l)}=\frac{\left|\boldsymbol{h}_{i}^{H} \boldsymbol{u}_{i}\right|}{\sum_{j=1, j \neq i}^{K}\left|\boldsymbol{h}_{i}^{H} \boldsymbol{u}_{k}\right|^{2}+1} .
$$

It has been shown that the SINR constraint can be transformed into semidefinite programming (SDP) or SOC form [24], and the corresponding beamforming problem can be solved via the standard interior point algorithm. However, the standard algorithm does not exploit the special structure of the problem, and may be computationally expensive. A number of efficient iterative algorithms have been proposed to solve the beamforming problem. In this section, we will combine these iterative algorithms and the general BC-MAC duality to solve the beamforming problem with multiple linear constraints. The methods discussed in this section are applicable to both SINR definitions (40) and (41).

\footnotetext{
${ }^{1}$ There are two reasons that we do not consider the MIMO BC scenario. 1) For the MIMO BC case, due to the multiple data streams, to impose the SINR constraint for each data stream is only appropriate when the independent encoding for each data stream is applied. In the previous work, the beamforming problems are considered only under the MISO scenario [15], [24]. 2) According to the definition (7), the SINR of the BC is neither convex nor concave with respect to $\boldsymbol{v}$ and $\boldsymbol{u}$ when $N_{t}>1$. Therefore, the MIMO optimization problem with SINR constraints is not a convex problem, and cannot be solved efficiently.
} 


\section{A. SINR Balancing Problem}

In this subsection, we apply the general BC-MAC duality in Section III to solve the SINR balancing problem with multiple linear constraints:

$$
\begin{gathered}
\max _{\boldsymbol{Q} \geq 0, \alpha \geq 0} \alpha \\
\text { subject to } \operatorname{SINR}_{i} \geq \alpha \gamma_{i}, \quad \forall i \\
\operatorname{tr}\left(\boldsymbol{Q} \boldsymbol{A}_{1}\right) \leq P_{1} \\
\operatorname{tr}\left(\boldsymbol{Q} \boldsymbol{A}_{2}\right) \leq P_{2} .
\end{gathered}
$$

Note that $\boldsymbol{Q}=\sum_{i=1}^{K} p_{i} \boldsymbol{u}_{i} \boldsymbol{u}_{i}^{H}$ in this case, and thus the problem can be viewed as a joint beamforming and power allocation problem. The SINR balancing problem for the BC has been studied in [15], [27]. However, due to the limitations of the conventional BC-MAC duality, previous results can be applied only to the case in which there is a single sum power constraint at the BS. Furthermore, the SINR balancing problem for the MAC with multiple constraints has been studied in [20], where it is shown that the multiple constraints can be completely decoupled. However, the decoupling property does not hold for the BC scenario.

To solve the problem (42), we first consider the following problem:

$$
\begin{aligned}
g_{\text {bal }}\left(\lambda_{1}, \lambda_{2}\right):= & \max _{\boldsymbol{u}_{i}, p_{i}, \alpha} \alpha \\
\text { subject to } & \operatorname{SINR}_{i} \geq \alpha \gamma_{i}, \forall i \\
& \lambda_{1} \operatorname{tr}\left(\boldsymbol{Q} \boldsymbol{A}_{1}\right)+\lambda_{2} \operatorname{tr}\left(\boldsymbol{Q} \boldsymbol{A}_{2}\right) \leq \lambda_{1} P_{1}+\lambda_{2} P_{2}
\end{aligned}
$$

where $\lambda_{1}$ and $\lambda_{2}$ are auxiliary variables. Similar to Proposition 3, we can prove that the optimal solution of the problem (43) is an upper bound on that of the problem (42). The problem (43) can be transformed into its dual MAC problem via the general BC-MAC duality and efficiently solved by the iterative algorithm in [15]. Moreover, the minimization problem $\min _{\lambda_{1}, \lambda_{2}} g_{\text {bal }}\left(\lambda_{1}, \lambda_{2}\right)$ can be solved through the subgradient algorithm or ellipsoid algorithm. The convexity of the function $g_{\text {bal }}\left(\lambda_{1}, \lambda_{2}\right)$ guarantees the convergence of the subgradient-based algorithm. Similar to the capacity region computation problem in Section IV-B, it can be proved that the algorithm converges to an optimal solution of the problem (42). 
Remark 5: The method to process multiple linear constraints in the SINR balancing problem is identical to that in the capacity computation problem. We first introduce an upper bound function, the solution of which can be obtained via the general BC-MAC duality. We next compute the minimum value of the upper bound function via a subgradient-based algorithm. Note that the iterative algorithm in [15] cannot be applied to the minimax duality case as the iterative algorithm requires the explicit expression of the noise covariance matrix. From this perspective, the general BC-MAC duality has broader application than the minimax duality.

\section{B. Power Balancing Problem}

In this subsection, we consider the power balancing problem with SINR constraints. Mathematically, the problem is formulated as follows:

$$
\begin{gathered}
\min _{\boldsymbol{Q} \geq 0, \alpha \geq 0} \alpha \\
\text { subject to } \operatorname{SINR}_{i} \geq \gamma_{i} \\
\operatorname{tr}\left(\boldsymbol{Q} \boldsymbol{A}_{1}\right) \leq \alpha P_{1} \\
\operatorname{tr}\left(\boldsymbol{Q} \boldsymbol{A}_{2}\right) \leq \alpha P_{2} .
\end{gathered}
$$

Since the problem (44) has multiple power constraints, the general BC-MAC duality cannot be applied directly. By introducing two auxiliary variables $\lambda_{1}$ and $\lambda_{2}$, we transform the problem (44) into the following single power constraint problem:

$$
\begin{aligned}
g_{\text {pow }}\left(\lambda_{1}, \lambda_{2}\right):= & \min _{\boldsymbol{Q} \geq 0, \alpha \geq 0} \alpha \\
\text { subject to } & \operatorname{SINR}_{i} \geq \gamma_{i} \\
& \lambda_{1} \operatorname{tr}\left(\boldsymbol{Q} \boldsymbol{A}_{1}\right)+\lambda_{2} \operatorname{tr}\left(\boldsymbol{Q} \boldsymbol{A}_{2}\right) \leq \alpha\left(\lambda_{1} P_{1}+\lambda_{2} P_{2}\right) .
\end{aligned}
$$

Similar to Proposition 3, the optimal solution of the problem (45) is a lower bound on that of the problem (44). Thanks to the general BC-MAC duality, the MIMO BC problem (45) is equivalent to its dual MAC problem as follows:

$$
\begin{array}{r}
\min _{\boldsymbol{Q} \geq 0} \sum_{i=1}^{K} \operatorname{tr}\left(\boldsymbol{Q}_{i}^{(m)}\right) \\
\text { subject to } \operatorname{SINR}_{i}^{(m)} \geq \gamma_{i}
\end{array}
$$


where the noise covariance of this dual MAC is $\lambda_{1} \boldsymbol{A}_{1}+\lambda_{2} \boldsymbol{A}_{2}$. By solving the problem (46) with the algorithm in [28], and utilizing the MAC-BC transmit covariance matrix transformation, the optimal solution of the problem (45) can be obtained. Next, we consider a maximization problem as follows:

$$
\max _{\lambda_{1} \geq 0, \lambda_{2} \geq 0} g_{\text {pow }}\left(\lambda_{1}, \lambda_{2}\right) .
$$

Similar to Proposition 5, we have the following result concerning the subgradient of the function $g_{\text {pow }}\left(\lambda_{1}, \lambda_{2}\right)$.

Proposition 7: The subgradient of the function $g_{\text {pow }}\left(\lambda_{1}, \lambda_{2}\right)$ at $\left[\tilde{\lambda}_{1}, \tilde{\lambda}_{2}\right]$ is $\left[\operatorname{tr}\left(\tilde{\boldsymbol{Q}} \boldsymbol{A}_{1}\right)-P_{1}, \operatorname{tr}\left(\tilde{\boldsymbol{Q}} \boldsymbol{A}_{2}\right)-\right.$ $P_{2}$ ], where $\tilde{\boldsymbol{Q}}$ is the optimal solution of the inner layer problem (45) with $\lambda_{1}=\tilde{\lambda}_{1}$ and $\lambda_{2}=\tilde{\lambda}_{2}$.

The proof of Proposition 7 is similar to that of Proposition 5, and thus is omitted here. With Proposition 7, the maximization problem (47) can be solved through the subgradient algorithm or the ellipsoid algorithm. Similar to Proposition 4, when the maximum of $g_{\text {pow }}\left(\lambda_{1}, \lambda_{2}\right)$ is achieved, the optimal solution of the problem (45) is equal to that of the problem (44)

Remark 6: The maximum per-antenna power constraint minimization problem was considered in [5], which is a special case of the problem (44). In [5], the problem is transformed into its minimax dual MAC problem, in which the noise covariance matrix of its dual MAC is an unknown variable. A subgradient-based iterative algorithm is developed therein to obtain its optimal noise covariance. However, since the noise covariance matrix appears in both the constraints and the objective function, it is difficult to have a routine method to obtain its subgradient in [5]. While, in contrast, due to the clear physical meaning of the variables $\lambda_{i}$ (Lagrange dual variables with respect to some constraints), the subgradient of the lower bound function $g_{\text {pow }}\left(\lambda_{1}, \lambda_{2}\right)$ can be readily obtained.

\section{NumericAl Results}

In this section, we present several numerical results to illustrate the effectiveness of the proposed algorithms. For simplicity, we consider a MIMO BC with $K=2, N_{t}=2, N_{r}=2$ for the capacity computation problem and with $K=2, N_{t}=2, N_{r}=1$ for the beamforming problem. The noise covariance matrix at each user is assumed to be an identity matrix. 


\section{A. Capacity Region of the MIMO BC}

In this example, we compute the capacity regions of the MIMO BC with a sum power constraint, a per-antenna power constraint and sum power plus per-antenna power constraints, separately. The sum power constraint is taken to be 10 , and the per-antenna power is taken to be 5 . For the sum power plus per-antenna power constraint case, the sum power constraint is 8 , and the per-antenna power constraint is 5. The channel matrices are chosen to be $\boldsymbol{H}_{1}=\left[\begin{array}{cc}1 & 0 \\ 0.2 & 0.6\end{array}\right]$ and $\boldsymbol{H}_{2}=\left[\begin{array}{cc}0.5 & 0 \\ 0.2 & 1\end{array}\right]$. For the case with a sum power constraint, the algorithm is similar to that in [6]. For the case with a per-antenna power constraint the subgradient-based iterative algorithm developed in Section IV is applied. For the sum power plus per-antenna power constraints case, two different algorithms are adopted. The first one is the subgradient-based iterative algorithm. The second algorithm is a heuristic algorithm, and is based on the result obtained in the case with a sum power constraint. With the sum power constraint solution, the transmit covariance matrix is normalized such that each antenna's power satisfies the per-antenna power constraint. The regions obtained by these algorithms are shown in Fig. 3. Since the heuristic algorithm obtains the suboptimal solution, the fourth line is just an achievable rate region of the MIMO BC with sum power plus per-antenna power constraints. Moreover, since the per-antenna power constraint is stricter than the sum power constraint, the capacity region of the case with a sum power constraint is larger than that of the case with a per-antenna power constraint.

\section{B. Weighted Sum Rate Maximization With Nonlinear Constraint}

This subsection is to present the simulation result of the Example 1 in Section IV-D. Suppose that $P$ in (36) is 100. The channel matrices are chosen to be $\boldsymbol{H}_{1}=\left[\begin{array}{cc}2 & 0 \\ 0.5 & 0.6\end{array}\right]$ and $\boldsymbol{H}_{2}=\left[\begin{array}{cc}0.3 & 0.2 \\ 0 & 1.5\end{array}\right]$. In Fig. 4 (a), the non-linear constraint function values are plotted versus the iteration steps. It can be observed that the non-linear transmit covariance constraint is satisfied when the optimal solution is achieved. In Fig. 2 (b), the achieved sum rates are plotted versus the iteration steps. The curve in Fig. 4 (b) is non-increasing, since the results in the former steps are obtained by solving the weighted sum rate problem with relaxed constraints. 


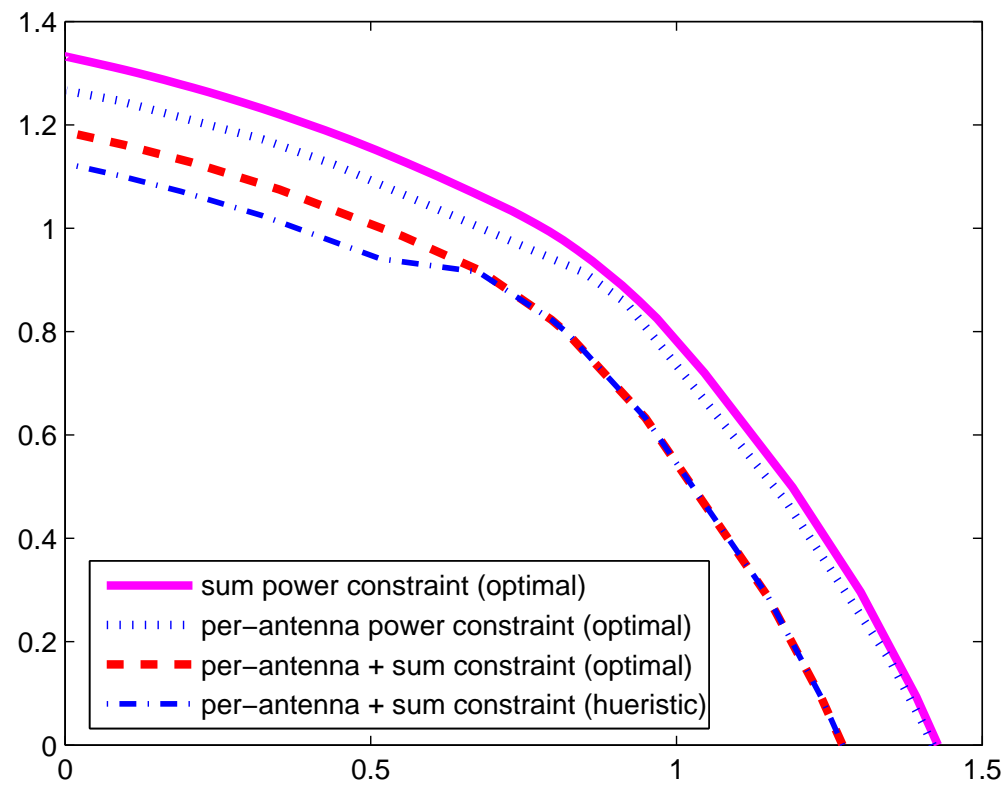

Fig. 3. The capacity regions for the MIMO BC with various power constraints.

\section{SINR Balancing With Multiple Linear Transmit Covariance Constraints}

In this example, we consider the SINR balancing problem with a per-antenna power constraint. We assume that each antenna's transmit power is subject to the constraint 5, and each user's target SINR is $\gamma_{i}=1$, for $i=1,2$. The channel matrix is chosen to be $\boldsymbol{H}_{1}=\left[\begin{array}{cc}1 & 0 \\ 0.5 & 0.6\end{array}\right]$ and $\boldsymbol{H}_{2}=\left[\begin{array}{cc}0.4 & 0 \\ 0.5 & 1.5\end{array}\right]$. The convergence behavior of the algorithm in Section V-A is shown in Fig. 5. The achieved SINR for each iteration is plotted in Fig. 5 (a). It can be observed that the curve in Fig. 5 (a) is non-increasing, and the achieved SINR for each iteration is greater than or equal to the final result. This is because that the optimal solution of the problem (43) is an upper bound on that of the original problem (42). The auxiliary variable values are plotted in Fig. 5 (b). It can be seen from this figure that only one constraint is active when the optimal solution is achieved. 


\section{CONCLUSIONS}

In this paper, we have established a general Gaussian BC-MAC duality, where the BC is subject to a general linear constraint and the MAC is subject to a weighted sum power constraint. This general BCMAC duality can be applied to solve the capacity computation and beamforming optimization problems with multiple convex linear/nonlinear constraints. The relationship between the new method and the previous minimax-duality based method has also been discussed. Moreover, it has been shown that, compared to the minimax duality the general BC-MAC duality offer greater flexibility for solving BC optimization problems. This new duality also generalizes the conventional Gaussian BC-MAC duality.

\section{APPENDIX}

A. Proof of Corollary 1: The corollary can be derived from Proposition 1 and the relationship between the SINR and the achievable rate (8). We will verify the corollary directly via covariance matrix transformation, as follows.

The achievable rate of the $i$ th user of the dual MAC can be written as

$$
\begin{aligned}
r_{i}^{(m)} & =\log \frac{\left|\boldsymbol{A}+\sum_{k=1}^{i} \boldsymbol{H}_{k}^{H} \boldsymbol{Q}_{k}^{(m)} \boldsymbol{H}_{k}\right|}{\left|\boldsymbol{A}+\sum_{k=1}^{i-1} \boldsymbol{H}_{k}^{H} \boldsymbol{Q}_{k}^{(m)} \boldsymbol{H}_{k}\right|} \\
& =\log \frac{\left|\boldsymbol{I}+\sum_{k=1}^{i} \boldsymbol{A}^{-1} \boldsymbol{H}_{k}^{H} \boldsymbol{Q}_{k}^{(m)} \boldsymbol{H}_{k}\right|}{\left|\boldsymbol{I}+\sum_{k=1}^{i-1} \boldsymbol{A}^{-1} \boldsymbol{H}_{k}^{H} \boldsymbol{Q}_{k}^{(m)} \boldsymbol{H}_{k}\right|} \\
& =\log \frac{\left|\boldsymbol{I}+\sum_{k=1}^{i}\left(\boldsymbol{H}_{k} \boldsymbol{A}^{-1 / 2}\right)^{H} \boldsymbol{Q}_{k}^{(m)} \boldsymbol{H}_{k} \boldsymbol{A}^{-1 / 2}\right|}{\left|\boldsymbol{I}+\sum_{k=1}^{i-1}\left(\boldsymbol{H}_{k} \boldsymbol{A}^{-1 / 2}\right)^{H} \boldsymbol{Q}_{k}^{(m)} \boldsymbol{H}_{k} \boldsymbol{A}^{-1 / 2}\right|}
\end{aligned}
$$

where the eigenvalue decomposition of $\boldsymbol{A}^{-1}$ is $\boldsymbol{U}^{H} \boldsymbol{\Lambda} \boldsymbol{U}$, and $\boldsymbol{A}^{-1 / 2}=\boldsymbol{U}^{H} \boldsymbol{\Lambda}^{1 / 2}$. According to (50), the MAC can be viewed as a virtual MAC with $\left(\boldsymbol{H}_{k} \boldsymbol{A}^{-1 / 2}\right)^{H}$ being its channel matrix and its noise covariance matrix being an identity matrix. By exploiting the BC-MAC covariance algorithm in [12], the achievable region of the virtual MAC is equal to the virtual $B C$ with $\boldsymbol{H}_{k} \boldsymbol{A}^{-1 / 2}$ being its channel matrix. Thus, the achievable rate of the $i$ user of the virtual $\mathrm{BC}$ can be written as

$$
r_{i}=\log \frac{\left|\boldsymbol{I}+\sum_{k=i}^{K} \boldsymbol{H}_{k} \boldsymbol{A}^{-1 / 2} \boldsymbol{Q}_{k}\left(\boldsymbol{H}_{k} \boldsymbol{A}^{-1 / 2}\right)^{H}\right|}{\left|\boldsymbol{I}+\sum_{k=i+1}^{K} \boldsymbol{H}_{k} \boldsymbol{A}^{-1 / 2} \boldsymbol{Q}_{k}\left(\boldsymbol{H}_{k} \boldsymbol{A}^{-1 / 2}\right)^{H}\right|}
$$

where $\sum_{k=1}^{K} \operatorname{tr}\left(\boldsymbol{Q}_{k}\right)=P$. By defining $\boldsymbol{Q}_{k}^{(b)}=\boldsymbol{A}^{-1 / 2} \boldsymbol{Q}_{k}\left(\boldsymbol{A}^{-1 / 2}\right)^{H}$, we have $\sum_{k=1}^{K} \operatorname{tr}\left(\boldsymbol{A} \boldsymbol{Q}_{k}^{(b)}\right)=P$. Thus, the achievable rate of the dual MAC with sum power constraint $P$ is also achievable for the primal 
$\mathrm{BC}$ with the constraint $\operatorname{tr}(\boldsymbol{A} \boldsymbol{Q}) \leq P$. Similarly, we can prove that the achievable rate of the primal $\mathrm{BC}$ with the constraint $\operatorname{tr}(\boldsymbol{A Q}) \leq P$ is also achievable for the dual MAC with the sum power constraint $P$. The proof follows.

B. Proof of Proposition 5: According to the definition of the subgradient, if $\left[s_{1}, s_{2}\right]$ is the subgradient of $g\left(\lambda_{1}, \lambda_{2}\right)$ at point $\left[\bar{\lambda}_{1}, \bar{\lambda}_{2}\right]$, then we have $g\left(\tilde{\lambda}_{1}, \tilde{\lambda}_{2}\right) \geq g\left(\bar{\lambda}_{1}, \bar{\lambda}_{2}\right)+\left[s_{1}, s_{2}\right] \cdot\left(\left[\tilde{\lambda}_{1}, \tilde{\lambda}_{2}\right]-\left[\bar{\lambda}_{1}, \bar{\lambda}_{2}\right]\right)^{H}$ for any $\left[\tilde{\lambda}_{1}, \tilde{\lambda}_{2}\right]$.

The Lagrange function of the problem (25) can be written as

$$
L(\boldsymbol{Q}, \lambda)=\sum_{i=1}^{K} w_{i} r_{i}-\lambda\left(\lambda_{1} \operatorname{tr}\left(\boldsymbol{Q} \boldsymbol{A}_{1}\right)+\lambda_{2} \operatorname{tr}\left(\boldsymbol{Q} \boldsymbol{A}_{2}\right)-\lambda_{1} P_{1}-\lambda_{2} P_{2}\right) .
$$

Thus, the corresponding dual problem is

$$
\min _{\lambda \geq 0} \max _{\boldsymbol{Q}} L\left(\boldsymbol{Q}, \lambda, \lambda_{1}, \lambda_{2}\right)
$$

We have

$$
\begin{aligned}
& g\left(\tilde{\lambda}_{1}, \tilde{\lambda}_{2}\right)-g\left(\bar{\lambda}_{1}, \bar{\lambda}_{2}\right) \\
& =\left.L(\boldsymbol{Q}, \lambda)\right|_{\boldsymbol{Q}=\tilde{\boldsymbol{Q}}, \lambda=\tilde{\lambda}} ^{\tilde{\lambda}_{1}, \tilde{\lambda}_{2}}-\left.L(\boldsymbol{Q}, \lambda)\right|_{\boldsymbol{Q}=\tilde{\boldsymbol{Q}}, \lambda=\bar{\lambda}} ^{\bar{\lambda}_{1}, \bar{\lambda}_{2}} \\
& \geq\left. L(\boldsymbol{Q}, \lambda)\right|_{\boldsymbol{Q}=\overline{\boldsymbol{Q}}, \lambda=\tilde{\lambda}} ^{\tilde{\lambda}_{1}, \tilde{\lambda}_{2}}-\left.L(\boldsymbol{Q}, \lambda)\right|_{\boldsymbol{Q}=\tilde{\boldsymbol{Q}}, \lambda=\bar{\lambda}} ^{\bar{\lambda}_{1}, \bar{\lambda}_{2}} \\
& =-\tilde{\lambda}\left(\tilde{\lambda}_{1}\left(\operatorname{tr}\left(\overline{\boldsymbol{Q}} \boldsymbol{A}_{1}\right)-P_{1}\right)+\tilde{\lambda}_{2}\left(\operatorname{tr}\left(\overline{\boldsymbol{Q}} \boldsymbol{A}_{2}\right)-P_{2}\right)\right)+\bar{\lambda}\left(\bar{\lambda}_{1}\left(\operatorname{tr}\left(\overline{\boldsymbol{Q}} \boldsymbol{A}_{1}\right)-P_{1}\right)+\bar{\lambda}_{2}\left(\operatorname{tr}\left(\overline{\boldsymbol{Q}} \boldsymbol{A}_{2}\right)-P_{2}\right)\right) \\
& =\left(\operatorname{tr}\left(\overline{\boldsymbol{Q}} \boldsymbol{A}_{1}\right)-P_{1}\right)\left(-\tilde{\lambda} \tilde{\lambda}_{1}+\bar{\lambda} \bar{\lambda}_{1}\right)+\left(\operatorname{tr}\left(\overline{\boldsymbol{Q}} \boldsymbol{A}_{2}\right)-P_{2}\right)\left(-\tilde{\lambda} \tilde{\lambda}_{2}+\bar{\lambda} \bar{\lambda}_{2}\right) \\
& =\left(\operatorname{tr}\left(\overline{\boldsymbol{Q}} \boldsymbol{A}_{1}\right)-P_{1}\right)\left(-\tilde{\lambda} \bar{\lambda}_{1}+\tilde{\lambda} \bar{\lambda}_{1}-\tilde{\lambda} \tilde{\lambda}_{1}+\bar{\lambda} \bar{\lambda}_{1}\right)+\left(\operatorname{tr}\left(\overline{\boldsymbol{Q}} \boldsymbol{A}_{2}\right)-P_{2}\right)\left(-\tilde{\lambda} \bar{\lambda}_{2}+\tilde{\lambda} \bar{\lambda}_{2}-\tilde{\lambda} \tilde{\lambda}_{2}+\bar{\lambda} \bar{\lambda}_{2}\right) \\
& =\left(\operatorname{tr}\left(\overline{\boldsymbol{Q}} \boldsymbol{A}_{1}\right)-P_{1}\right)\left(-\tilde{\lambda} \tilde{\lambda}_{1}+\tilde{\lambda} \bar{\lambda}_{1}\right)+\left(\operatorname{tr}\left(\overline{\boldsymbol{Q}} \boldsymbol{A}_{1}\right)-P_{1}\right)\left(\bar{\lambda} \bar{\lambda}_{1}-\tilde{\lambda} \bar{\lambda}_{1}\right) \\
& +\left(\operatorname{tr}\left(\overline{\boldsymbol{Q}} \boldsymbol{A}_{2}\right)-P_{2}\right)\left(-\tilde{\lambda} \tilde{\lambda}_{2}+\tilde{\lambda} \bar{\lambda}_{2}\right)+\left(\operatorname{tr}\left(\overline{\boldsymbol{Q}} \boldsymbol{A}_{2}\right)-P_{2}\right)\left(\bar{\lambda} \bar{\lambda}_{2}-\tilde{\lambda} \bar{\lambda}_{2}\right) \\
& =\tilde{\lambda}\left[P_{1}-\operatorname{tr}\left(\overline{\boldsymbol{Q}} \boldsymbol{A}_{1}\right), P_{2}-\operatorname{tr}\left(\overline{\boldsymbol{Q}} \boldsymbol{A}_{2}\right)\right] \cdot\left[\tilde{\lambda}_{1}-\bar{\lambda}_{1}, \tilde{\lambda}_{2}-\bar{\lambda}_{2}\right]^{H}
\end{aligned}
$$

where (61) is due to $\left(\operatorname{tr}\left(\overline{\boldsymbol{Q}} \boldsymbol{A}_{1}\right)-P_{1}\right) \bar{\lambda}_{1}+\left(\operatorname{tr}\left(\overline{\boldsymbol{Q}} \boldsymbol{A}_{2}\right)-P_{2}\right) \bar{\lambda}_{2}=0$. The proof follows. 
C. Proof of Proposition 6: Suppose that $Q^{*}$ is the optimal solution of the problem (36). The KKT conditions of the problem (36) can be written as

$$
\begin{aligned}
& \left.\frac{\partial \sum_{i=1}^{K} w_{i} r_{i}}{\partial \boldsymbol{Q}_{i}}\right|_{\boldsymbol{Q}_{i}=\boldsymbol{Q}_{i}^{*}}=\left.\lambda f^{\prime}\left(\boldsymbol{Q}_{i}\right)\right|_{\boldsymbol{Q}_{i}=\boldsymbol{Q}_{i}^{*}}+\boldsymbol{\Psi}_{i}, \forall i \\
& \lambda f\left(\boldsymbol{Q}^{*}\right)=0 .
\end{aligned}
$$

Note that $\boldsymbol{Q}=\sum_{i=1}^{K} \boldsymbol{Q}_{i}$, and thus we have $f^{\prime}\left(\boldsymbol{Q}_{i}\right)=f^{\prime}(\boldsymbol{Q}) \frac{\partial \boldsymbol{Q}}{\partial \boldsymbol{Q}_{i}}=f^{\prime}(\boldsymbol{Q})$. Now, let us consider the linear constraint problem

$$
\max _{Q} \sum_{i=1}^{K} w_{i} r_{i}
$$

$$
\text { subject to } \operatorname{tr}(\boldsymbol{A Q}) \leq 0
$$

where $\boldsymbol{A}=\left.f^{\prime}(\boldsymbol{Q})\right|_{\boldsymbol{Q}=Q^{*}}$. The KKT conditions for the problem (64) are

$$
\begin{aligned}
& \left.\frac{\partial \sum_{i=1}^{K} w_{i} r_{i}}{\partial \boldsymbol{Q}_{i}}\right|_{\boldsymbol{Q}_{i}=\boldsymbol{Q}_{i}^{*}}=\left.\lambda f^{\prime}\left(\boldsymbol{Q}_{i}\right)\right|_{\boldsymbol{Q}_{i}=\boldsymbol{Q}_{i}^{*}}+\boldsymbol{\Psi}_{i}, \forall i \\
& \lambda f\left(\boldsymbol{Q}^{*}\right)=0 .
\end{aligned}
$$

It is easy to observe that $Q^{*}$ satisfies the KKT conditions of the problem (64). Combining this with Proposition 2, we can conclude that $Q^{*}$ is the optimal solution of problem (64). The proof follows.

\section{REFERENCES}

[1] P. Rashid-Farrokhi, K. J. R. Liu, and L. Tassiulas, “Transmit beamforming and power control for cellular wireless systems,” IEEE J. Select. Areas Commun., vol. 16, no. 8, pp. 1437-1449, Oct. 1998.

[2] F. Rashid-Farrokhi, L. Tassiulas, and K. J. R. Liu, "Joint optimal power control and beamforming in wireless networks using antenna arrays," IEEE Trans. Commun., vol. 46, no. 10, pp. 1313-1324, Oct. 1998.

[3] N. Jindal, W. Rhee, S. Vishwanath, S. A. Jafar, and A. Goldsmith, "Sum power iterative water-filling for multi-antenna Gaussian broadcast channels," IEEE Trans. Inform. Theory, vol. 51, no. 4, pp. 1570-1580, Apr. 2005.

[4] W. Yu, "Sum-capacity computation for the Gaussian vector broadcast channel via dual decomposition," IEEE Trans. Inform. Theory, vol. 52, no. 2, pp. 754-759, Feb. 2006.

[5] W. Yu and T. Lan, "Transmitter optimization for the multi-antenna downlink with per-antenna power constraints," IEEE Trans. Signal Processing, vol. 55, no. 6, pp. 2646-2660, June 2007.

[6] M. Mohseni, R. Zhang, and J. M. Cioffi, "Optimized transmission for fading multiple-access and broadcast channels with multiple antennas," IEEE J. Select. Areas Commun., vol. 24, no. 8, pp. 1627-1639, Aug. 2006.

[7] D. Tse and P. Viswanath, "Downlink-uplink duality and effective bandwidths," in Proc. IEEE Int. Symp. Inf. Theory (ISIT), Lausanne, Switzerland, July 2002. 
[8] P. Viswanath and D. N. C. Tse, "Sum capacity of the vector Gaussian broadcast channel and uplink-downlink duality," IEEE Trans. Inform. Theory, vol. 49, no. 8, pp. 1912-1921, Aug. 2003.

[9] N. Jindal, S. Vishwanath, and A. Goldsmith, "On the duality of Gaussian multiple-access and broadcast channels," IEEE Trans. Inform. Theory, vol. 50, no. 5, pp. 768-783, May 2004.

[10] W. Yu, "Uplink-downlink duality via minimax duality," IEEE Trans. Inform. Theory, vol. 52, no. 2, pp. 361-374, Feb. 2006.

[11] W. Yu and J. Cioffi, "Sum capacity of Gaussian vector broadcast channels," IEEE Trans. Inform. Theory, vol. 50, no. 9, pp. 1875-1892, Sept. 2004.

[12] S. Vishwanath, N. Jindal, and A. Goldsmith, "Duality, achievable rates, and sum-rate capacity of Gaussian MIMO broadcast channels," IEEE Trans. Inform. Theory, vol. 49, no. 10, pp. 2658-2668, Oct. 2003.

[13] I. E. Telatar, "Capacity of multi-antenna Gaussian channels," European Trans. on Telecomm., vol. 10, no. 6, pp. 585-595, Oct. 1999.

[14] E. Visotsky and U. Madhow, "Optimum beamforming using transmit antenna arrays," in Proc. IEEE Veh. Tech. Conf., Houston, TX, USA, May 1999, pp. 851-856.

[15] M. Schubert and H. Boche, "Solution of the multiuser downlink beamforming problem with individual SINR constraints," IEEE Trans. Veh. Technol., vol. 53, no. 1, pp. 18-28, Jan. 2004.

[16] M. H. M. Costa, "Writing on dirty paper," IEEE Trans. Inform. Theory, vol. 29, no. 3, pp. 439-441, May 1983.

[17] H. Weingarten, Y. Steinberg, and S. Shamai, "The capacity region of the Gaussian multiple-input multiple-output broadcast channel," IEEE Trans. Inform. Theory, vol. 52, no. 9, pp. 3936-64, Sept. 2006.

[18] W. Yu, W. Rhee, S. Boyd, and J. M. Cioffi, "Iterative water-filling for Gaussian vector multiple-access channels," IEEE Trans. Inform. Theory, vol. 50, no. 1, pp. 145-152, Jan. 2004.

[19] D. Tse and P. Viswanath, Fundamentals of Wireless Communications. Cambridge University Press, 2005.

[20] L. Zhang, Y.-C. Liang, and Y. Xin, "Joint beamforming and power allocation for multiple access channels in cognitive radio networks," IEEE J. Select. Areas Commun., vol. 26, no. 1, pp. 38-51, Jan. 2008.

[21] R. Zhang and Y.-C. Liang, "Exploiting multi-antennas for opportunistic spectrum sharing in cognitive radio networks," IEEE $J$. Select. Topics in Signal Processing, vol. 2, no. 1, pp. 88-102, Feb. 2008.

[22] L. Zhang, Y. Xin, and Y.-C. Liang, "Weighted sum rate optimization for cognitive radio mimo broadcast channels," in IEEE international conference on communications, Beijing, China, 19-23, May, 2008, pp. 3679-3683.

[23] — - "A weighted sum rate optimization for cognitive radio mimo broadcast channels," IEEE Trans. Wireless Commun., submitted for publication, Apri., 2008. [Online] Available: http://arxiv.org/abs/0804.3453v1.

[24] A. Wiesel, Y. C. Eldar, and S. Shamai, "Linear precoding via conic optimization for fixed MIMO receivers," IEEE Trans. Signal Processing, vol. 54, no. 1, pp. 161-176, Jan. 2006.

[25] S. Boyd and L. Vandenberghe, Convex Optimization. Cambridge, UK: Cambridge University Press, 2004.

[26] S. Boyd, L. Xiao, and A. Mutapcic, "Subgradient methods," 2003. [Online]. Available: http://mit.edu/6.976/www/notes/subgrad \{-\} method.pdf.

[27] W. Yang and G. Xu, "Optimal downlink power assignment for smart antenna systems," in IEEE Int. Conf. Acoust., Speech, and Signal Proc. (ICASSP), Seattle, Washington, USA, May 10-12, 1998.

[28] M. Schubert and H. Boche, "Iterative multiuser uplink and downlink beamforming under SINR constraints," IEEE Trans. Signal Processing, vol. 53, no. 7, pp. 2324-2334, July 2005. 


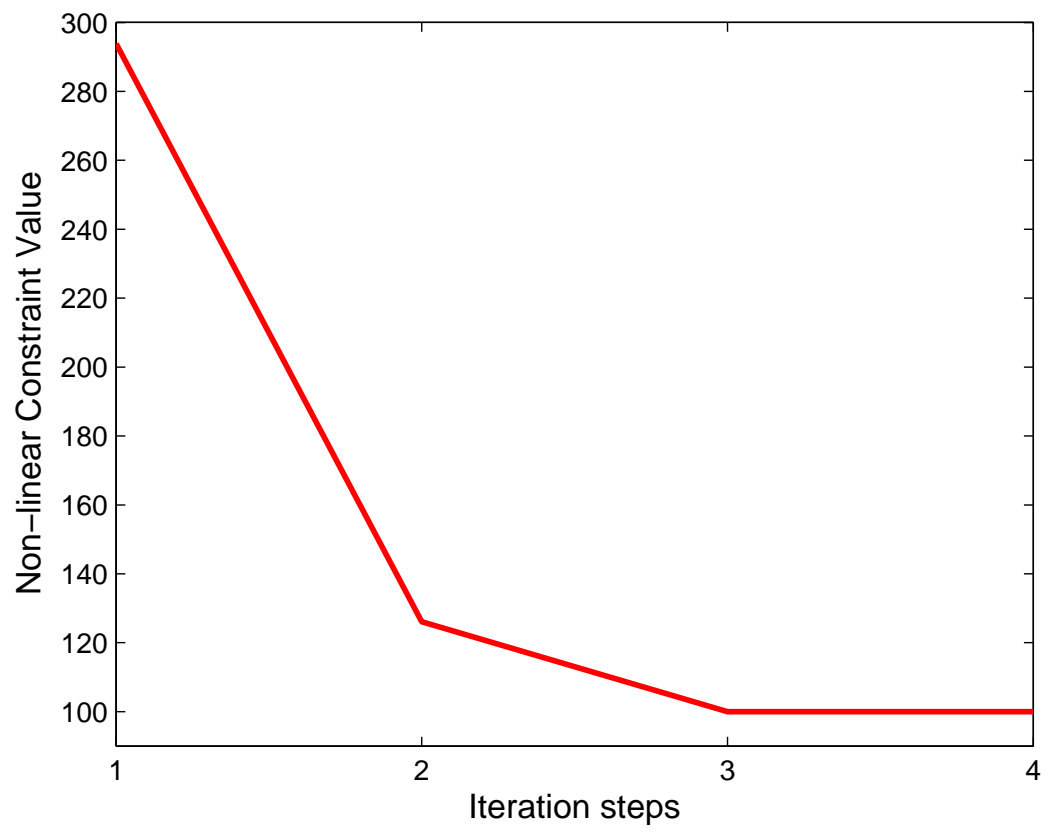

(a) The value of $\left(\operatorname{tr}\left(\boldsymbol{Q} \boldsymbol{A}_{1}\right)\right)^{2}+\left(\operatorname{tr}\left(\boldsymbol{Q} \boldsymbol{A}_{2}\right)\right)^{2}$ versus the iteration steps.

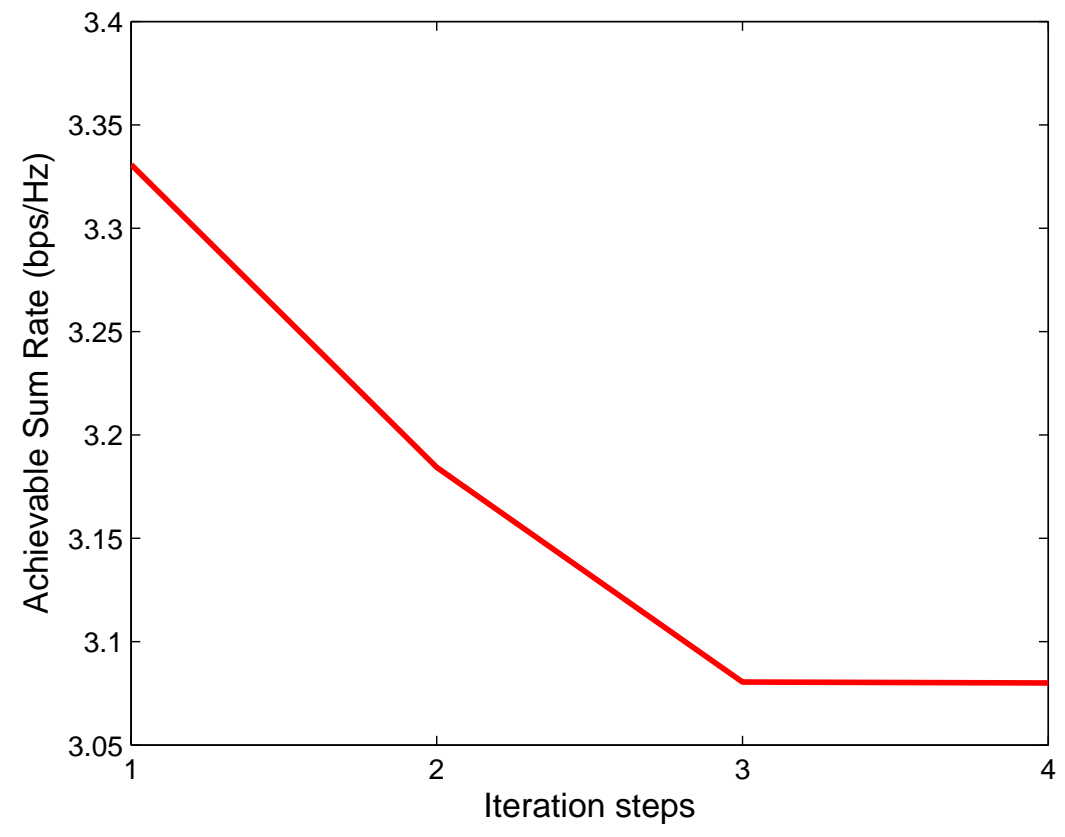

(b) Achieved sum rate versus the iteration steps.

Fig. 4. The convergence behavior of the subgradient-based algorithm for the weighted sum rate maximization problem with a non-linear constraint. 


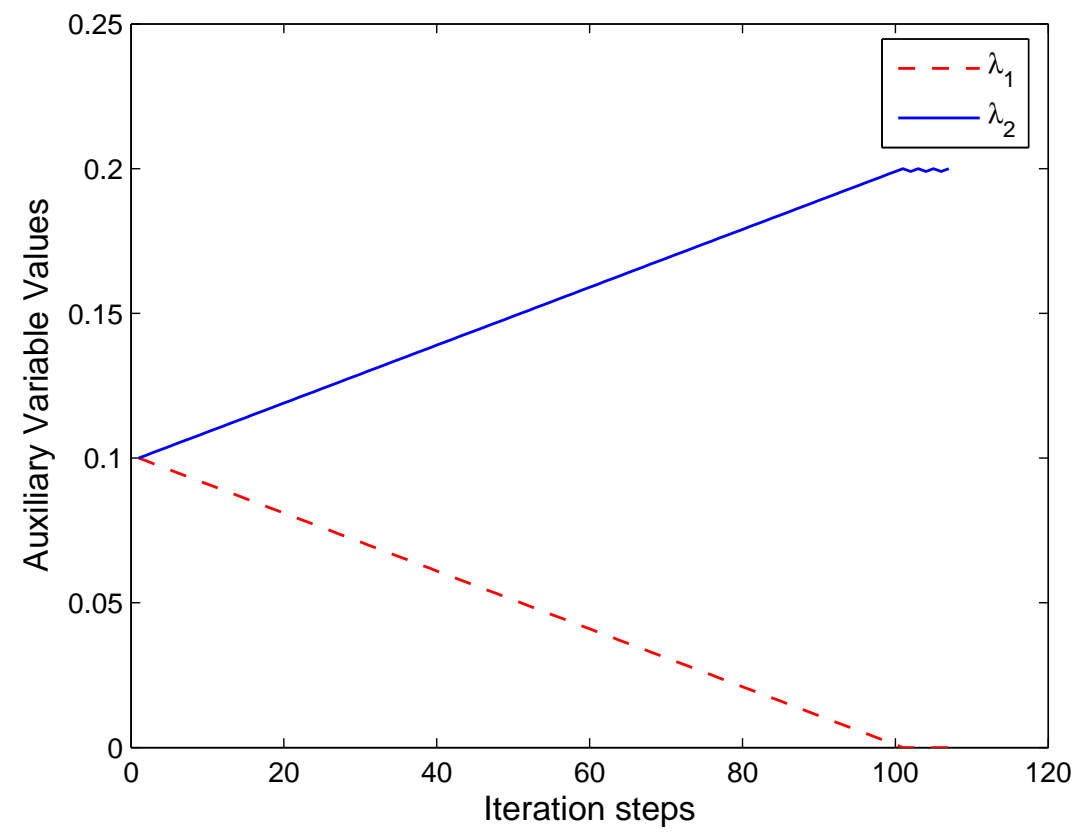

(a) Auxiliary variable values versus the iteration steps.

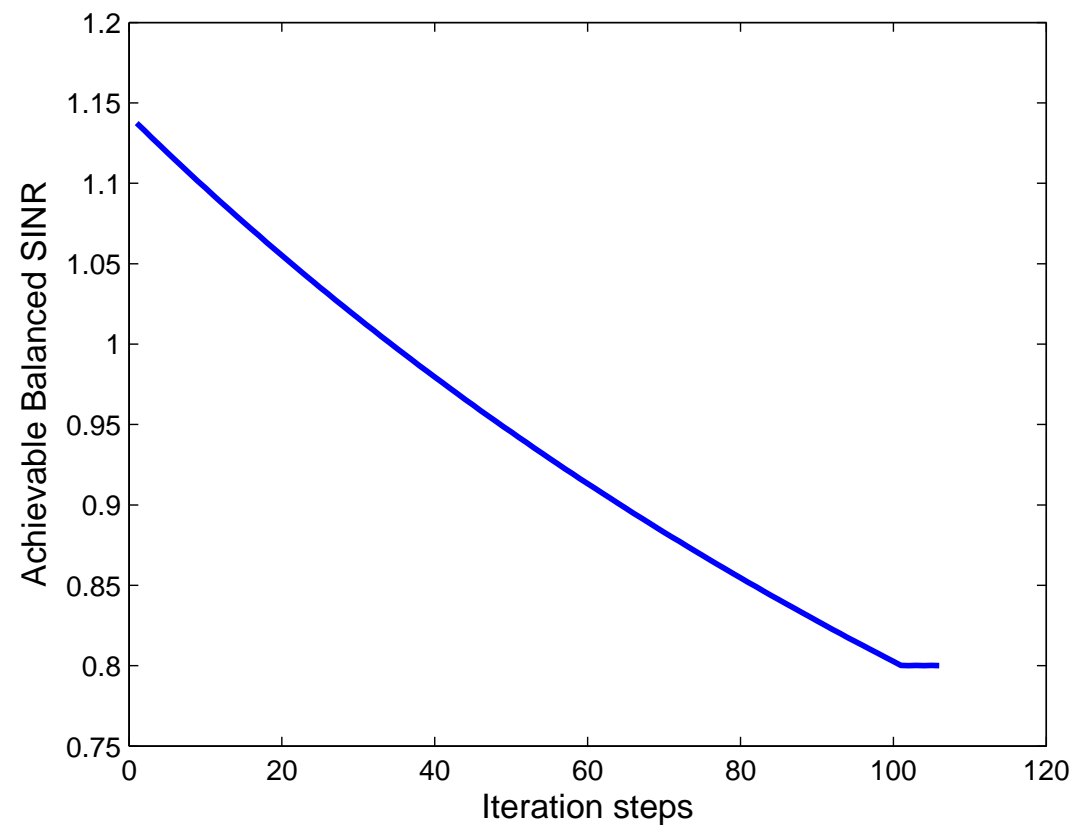

(b) Achieved balanced SINR versus the iteration steps.

Fig. 5. The convergence behavior of the subgradient-based algorithm for the SINR balancing problem with per-antenna power constraint. 